\title{
Semiconductor Saturable Absorber Mirrors (SESAM's) for Femtosecond to Nanosecond Pulse Generation in Solid-State Lasers
}

\author{
Ursula Keller, Member, IEEE, Kurt J. Weingarten, Member, IEEE, Franz X. Kärtner, Daniel Kopf, Bernd Braun, \\ Isabella D. Jung, Regula Fluck, Clemens Hönninger, Nicolai Matuschek, and Juerg Aus der Au
}

(Invited Paper)

\begin{abstract}
Intracavity semiconductor saturable absorber mirrors (SESAM's) offer unique and exciting possibilities for passively pulsed solid-state laser systems, extending from $Q$-switched pulses in the nanosecond and picosecond regime to mode-locked pulses from 10's of picoseconds to sub-10 fs. This paper reviews the design requirements of SESAM's for stable pulse generation in both the mode-locked and $Q$-switched regime. The combination of device structure and material parameters for SESAM's provide sufficient design freedom to choose key parameters such as recovery time, saturation intensity, and saturation fluence, in a compact structure with low insertion loss. We have been able to demonstrate, for example, passive modelocking (with no $Q$ switching) using an intracavity saturable absorber in solid-state lasers with long upper state lifetimes (e.g., 1- $\mu$ m neodymium transitions), Kerr lens modelocking assisted with pulsewidths as short as 6.5 fs from a Ti:sapphire laser-the shortest pulses ever produced directly out of a laser without any external pulse compression, and passive $Q$-switching with pulses as short as $56 \mathrm{ps}$ - the shortest pulses ever produced directly from a $Q$ switched solid-state laser. Diode-pumping of such lasers is leading to practical, real-world ultrafast sources, and we will review results on diode-pumped $\mathrm{Cr}$ :LiSAF, Nd:glass, Yb:YAG, Nd:YAG, Nd:YLF, Nd:LSB, and Nd:YVO . $^{.}$
\end{abstract}

\section{HISTORICAL BACKGROUND AND INTRODUCTION}

\section{A. Semiconductor Saturable Absorbers for Solid-State Lasers}

$\mathbf{T}$ THE use of saturable absorbers in solid-state lasers is practically as old as the solid-state laser itself [1]-[3]. However, it was believed that pure, continuous-wave (CW) modelocking could not be achieved using saturable absorbers with solid-state lasers such as Nd:glass, Nd:YAG, or Nd:YLF with long upper state lifetimes (i.e., $>100 \mu \mathrm{s}$ ) without $Q$ switching or $Q$-switched mode-locked behavior (Fig. 1). This limitation was mostly due to the parameter ranges of available saturable absorbers [4]. However, the advent of bandgap engineering and modern semiconductor growth technology has allowed for saturable absorbers with accurate control of the device parameters such as absorption wavelength, saturation energy, and recovery time, and we have been able to demonstrate pure passive $Q$-switching, pure $\mathrm{CW}$ modelocking

Manuscript received September 24, 1996; revised January 9, 1997.

The authors are with the Institute of Quantum Electronics, Swiss Federal Institute of Technology (ETH), ETH-Hönggerberg HPT, CH-8093 Zürich, Switzerland.

Publisher Item Identifier S 1077-260X(96)09675-X.

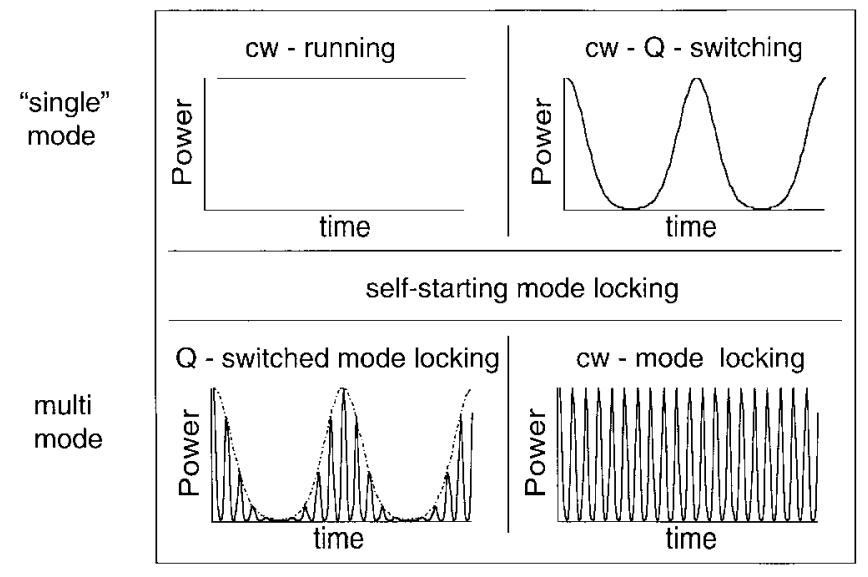

Fig. 1. Different modes of operation of a laser with a saturable absorber. CW $Q$-switching typically occurs with much longer pulses and lower pulse repetition rates than $\mathrm{CW}$ mode-locking.

or, if desired, $Q$-switched modelocking behavior [5]-[9]. In addition, semiconductor absorbers have an intrinsic bitemporal impulse response (Fig. 2): intraband carrier-carrier scattering and thermalization processes which are in the order of 10 to $100 \mathrm{fs}$ as well as interband trapping and recombination processes which can be in the order of picoseconds to nanoseconds depending on the growth parameters [10], [11]. As we will discuss, the faster saturable absorption plays an important role in stabilizing femtosecond lasers, while the slower response is important for starting the pulse formation process and for pulse forming in lasers with pulsewidths of picoseconds or longer.

Many other classes of laser can be passively mode-locked with saturable absorbers. Previously, semiconductor saturable absorbers have been successfully used to mode-locked semiconductor diode lasers, where the recovery time was reduced by damage induced either during the aging process [12], by proton bombardment [13], or by multiple quantum wells [14]. More recently, both bulk and multiple quantum-well semiconductor saturable absorbers have been used to mode-lock color center lasers [15]. In both cases, the upper state lifetime of the laser medium is in the nanosecond regime, which strongly reduces the tendency for self- $Q$-switching instabilities (discussed further in Section II). This is not the case for 


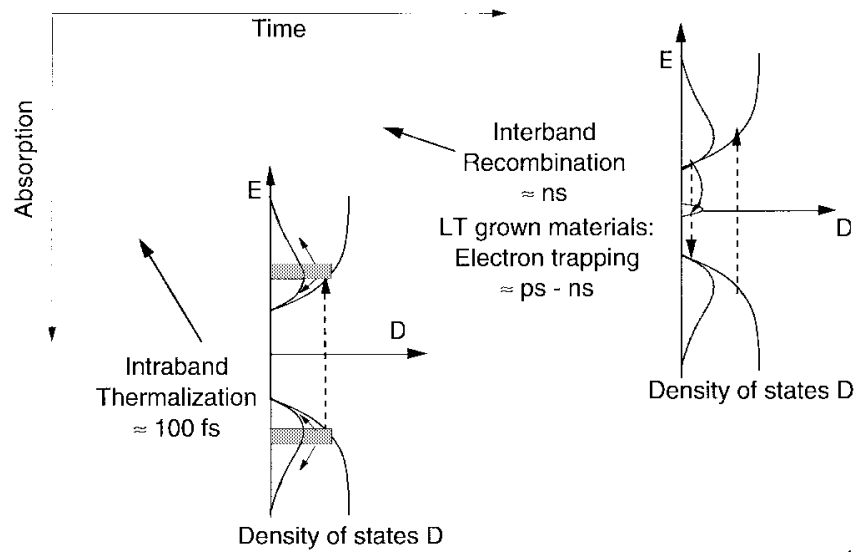

Fig. 2. A measured impulse response typical for a semiconductor saturable absorber. The optical nonlinearity is based on absorption bleaching.

most other solid-state lasers with an upper state laser lifetime in the microsecond to millisecond regime. First results with SESAM's in solid-state lasers were reported in 1990, and they were initially used in nonlinear coupled cavities [16]-[21], a technique termed RPM (resonant passive mode-locking). This paper was motivated by previously demonstrated soliton lasers [22] and APM (additive pulse mode-locking) lasers [23]-[25], where a nonlinear phase shift in a fiber inside a coupled cavity provided an effective saturable absorption. Most uses of coupled cavity techniques have been supplanted by intracavity saturable absorber techniques based on Kerr lens mode-locking (KLM) [26] and SESAM's [5], due to their more inherent simplicity. In 1992, we demonstrated a stable, purely CW-mode-locked Nd:YLF and Nd:YAG laser using an intracavity SESAM design, referred to as the antiresonant Fabry-Perot saturable absorber (A-FPSA) [5]. Since then, many new SESAM designs have been developed (see Section III) that provide stable pulse generation for a variety of solid-state lasers.

\section{B. Mode-Locking Mechanism for Solid-State Lasers: Fast- Saturable-Absorber Mode-Locking or Soliton Mode-Locking}

Passive mode-locking mechanisms are well-explained by three fundamental models: slow saturable absorber modelocking with dynamic gain saturation [27], [28] [Fig. 3(a)], fast saturable absorber mode-locking [29], [30] [Fig. 3(b)] and soliton mode-locking [31]-[33] [Fig. 3(c)]. In the first two cases, a short net-gain window forms and stabilizes an ultrashort pulse. This net-gain window also forms the minimal stability requirement, i.e., the net loss immediately before and after the pulse defines its extent. However, in soliton modelocking, where the pulse formation is dominated by the balance of group velocity dispersion (GVD) and self-phase modulation (SPM), we have shown that the net-gain window can remain open for more than ten times longer than the ultrashort pulse, depending on the specific laser parameters [32]. In this case, the slower saturable absorber only stabilizes the soliton and starts the pulse formation process.

Until the end of the 1980's, ultrashort pulse generation was dominated by dye lasers, where mode-locking was based on a balanced saturation of both gain and loss, opening a steady-
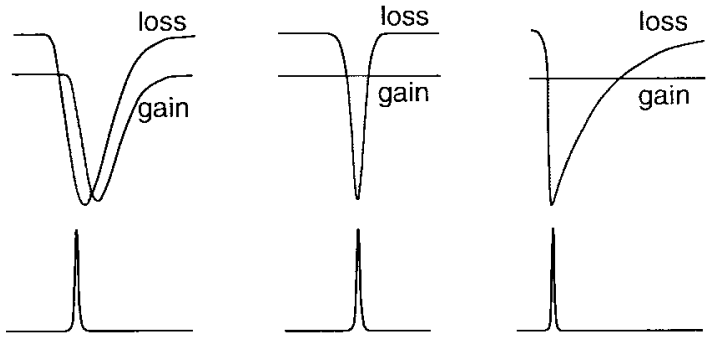

Fig. 3. The three fundamental passive mode-locking models: (a) passive mode-locking with a slow saturable absorber and dynamic gain saturation [27], [28], (b) fast absorber mode-locking [29], [30], and (c) soliton mode-locking [31]-[33]

state net gain window as short as the pulse duration [Fig. 3(a)] (the slow-absorber with dynamic gain saturation model [27], [28]). Pulses as short as $27 \mathrm{fs}$ with an average power of $\approx 10 \mathrm{~mW}$ were generated [34]. Shorter pulse durations to 6 fs were achieved through additional amplification and fibergrating pulse compression, although at much lower repetition rates [35].

The situation changed with the development and commercialization of the Ti:sapphire laser [36], which has a gainbandwidth large enough to support ultrashort pulse generation. However, existing mode-locking techniques were inadequate because of the much longer upper state lifetime and the smaller gain cross section of this laser, which results in negligible pulse-to-pulse dynamic gain saturation. Initially it was assumed that a fast saturable absorber would be required to generate ultrashort pulses [Fig. 3(b)]. Such a fast saturable absorber was discovered [26] and its physical mechanism described as Kerr lens mode-locking (KLM) [19], [37], [38], where strong self-focusing of the laser beam combined with either a hard aperture or a "soft" gain aperture is used to produce a self amplitude modulation, i.e., an equivalent fast saturable absorber. Since then, significant efforts have been directed toward optimizing KLM for shorter pulse generation, with the current results standing at around 8 fs [39]-[41] directly from the laser. Using a broad-band intracavity SESAM device in addition to KLM and higher order dispersion compensation [42], [43] we recently generated pulses as short as 6.5 fs [Fig. 12(b)] directly out of a Ti:sapphire laser with $200 \mathrm{~mW}$ average output power at a pulse repetition rate of $\approx 85 \mathrm{MHz}$ [44]. External pulse compression techniques based on fibergrating pulse compressors have been used to further reduce the pulse duration from a Ti:sapphire laser to $\approx 5$ fs at a center wavelength of $\approx 800 \mathrm{~nm}$ [45], [46]. These are currently the shortest optical pulses ever generated.

Besides the tremendous success of KLM, there are some significant limitations for practical or "real-world" ultrafast lasers. First, the cavity is typically operated near one end of its stability range, where the Kerr-lens-induced change of the beam diameter is large enough to sustain mode-locking. This results in a requirement for critical cavity alignment where mirrors and laser crystal have to be positioned to an accuracy of several hundred microns typically. Additionally, the self-focusing required for KLM imposes limitations on the cavity design and leads to strong space-time coupling of the pulses in the laser crystal that results in complex laser 
dynamics [47], [48]. Once the cavity is correctly aligned, KLM can be very stable and under certain conditions even selfstarting [49], [50]. However, self-starting KLM lasers in the sub-50-fs regime have not yet been demonstrated without any additional starting mechanisms as for example a SESAM. This is not surprising, since in a 10-fs Ti:sapphire laser with a 100 $\mathrm{MHz}$ repetition rate, the peak power changes by six orders of magnitude when the laser switches from CW to pulsed operation. Therefore, nonlinear effects that are still effective in the sub-10-fs regime are typically too small to initiate modelocking in the $\mathrm{CW}$-operation regime. In contrast, if self-starting is optimized, KLM tends to saturate in the ultrashort pulse regime or the large SPM will drive the laser unstable.

However, we have shown that a novel mode-locking technique, which we term soliton mode-locking [31]-[33], [51], addresses many of these issues. In soliton mode-locking, the pulse shaping is done solely by soliton formation, i.e., the balance of GVD and SPM at steady state, with no additional requirements on the cavity stability regime. An additional loss mechanism, such as a saturable absorber [31], [33], or an acousto-optic mode-locker [51], [52], is necessary to start the mode-locking process and to stabilize the soliton.

This can be explained as follows. The soliton loses energy due to gain dispersion and losses in the cavity. Gain dispersion and losses can be treated as perturbation to the nonlinear Schrödinger equation for which a soliton is a stable solution [51]. This lost energy, called continuum in soliton perturbation theory [53], is initially contained in a low intensity background pulse, which experiences negligible bandwidth broadening from SPM, but spreads in time due to GVD. This continuum experiences a higher gain compared to the soliton, because it only sees the gain at line center (while the soliton sees an effectively lower average gain due to its larger bandwidth). After a sufficient build-up time, the continuum would actually grow until it reaches an effective lasing threshold, destabilizing the soliton. However, we can stabilize the soliton by introducing a "slow" saturable absorber into the cavity. This slow absorber adds sufficient additional loss so that the continuum no longer reaches threshold, but with negligible increased loss for the short soliton pulse.

Depending on the specific laser parameters such as gain dispersion, small signal gain, and negative dispersion, a "slow" saturable absorber can stabilize a soliton with a response time of more than ten times longer than the steady-state soliton pulsewidth [Fig. 3(c)]. High-dynamic range autocorrelation measurements have shown ideal transform-limited soliton pulses over more than six orders of magnitude, even though the net gain window is open much longer than the pulse duration [32], [54], [55]. Due to the slow saturable absorber, the soliton undergoes an efficient pulse cleaning mechanism [33]. In each round-trip, the front part of the soliton is absorbed which delays the soliton with respect to the continuum.

In contrast to KLM, soliton mode-locking is obtained over the full cavity stability regime, and pulses as short as $13 \mathrm{fs}$ have been generated currently with a purely soliton-modelocked Ti:sapphire laser using a broad-band SESAM [33], [56]. Soliton mode-locking decouples SPM and self-amplitude modulation, potentially allowing for independent optimization.
We justify the introduction of a new name for this modelocking process because previously soliton effects were only considered to lead to a moderate additional pulsewidth reduction of up to a factor of 2 , but the stabilization was still achieved by a short net gain window as discussed for CPM dye [57]-[60] and for KLM Ti:sapphire lasers [61], [62].

\section{Design Criteria for a Saturable Absorber}

First we consider the basic design parameters of a general saturable absorber. These consist of the saturation intensity $I_{\text {sat }}$ and saturation fluence $E_{\text {sat }}$, which will be seen to influence the mode-locking build-up and the pulse stability with respect to self- $Q$-switching. In addition, the recovery time of the saturable absorber determines the dominant mode-locking mechanism, which is either based on fast saturable absorber mode-locking [Fig. 3(b)] in the positive or negative dispersion regime, or soliton mode-locking [Fig. 3(c)], which operates solely in the negative dispersion regime. For solid-state lasers we can neglect slow saturable absorber mode-locking as shown in Fig. 3(a), because no significant dynamic gain saturation is taking place due to the long upper state lifetime of the laser. When the recovery time of the absorber is on the order of or even larger than the laser's cavity round-trip time, the laser will tend to operate in the pure CW-Q-switching regime (Fig. 1).

In addition, the nonsaturable losses of a saturable absorber need to be small, because we typically only couple a few percent out of a CW mode-locked solid-state laser. As the nonsaturable losses increase, the laser becomes less efficient and operates fewer times over threshold, which increases the tendency for instabilities [see (4) and (6) below] such as $Q$-switched mode-locked behavior.

Fig. 4 shows the typical saturation behavior for an absorber on a mirror. Initially, the pulses are formed by noise fluctuations in the laser, and the saturation amount at this early stage is dominated by the $\mathrm{CW}$ intensity $I$ incident on the absorber [Fig. 4(a)]. In general, we can assume that the saturable absorber is barely bleached (i.e., $I \ll I_{\text {sat }}$ ) at $\mathrm{CW}$ intensity, because if the absorber were fully bleached at this intensity, there would be insufficient further modulation to drive the pulse forming process.

The saturation intensity $I_{\text {sat }}$ is given by

$$
I_{\text {sat }}=\frac{h \nu}{\sigma_{A} T_{A}}
$$

where $h \nu$ is the photon energy, $\sigma_{A}$ the absorption cross section and $T_{A}$ the absorber recovery time. It is important to note that the absorption cross section is effectively a material parameter. The absorption coefficient $\alpha$ of the material is then given by

$$
\alpha=\sigma_{A} N_{D}
$$

where $N_{D}$ is the density of absorber atoms or the density of states in semiconductors, for example.

Referring again to Fig. 4(a), the slope $d R / d I$ at around $I \approx 0$ determines the mode-locking build-up time under certain approximations [9] can be written as

$$
T_{\text {build-up }} \propto \frac{1}{\left.\frac{d R}{d I}\right|_{I \approx 0} I} .
$$




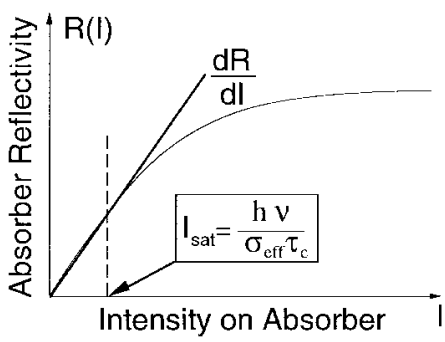

(a)

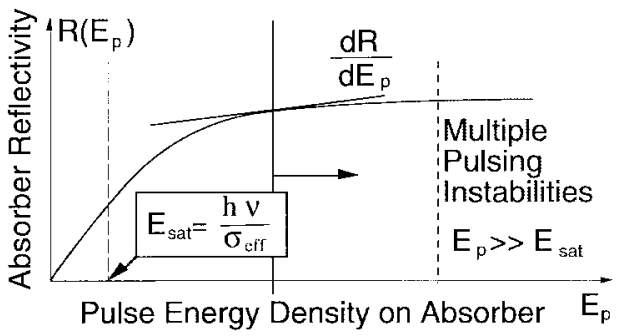

(b)

Fig. 4. Nonlinear reflectivity change of a saturable absorber mirror due to absorption bleaching with the (a) CW intensity and (b) short pulses. $I_{\text {sat }}$ is the saturation intensity, $E_{\text {sat }}$ is the saturation fluence, $I$ is the $\mathrm{CW}$ intensity, and $E_{p}$ is the pulse energy density incident on the saturable absorber.

As expected, the build-up time is inversely proportional to this slope. This follows directly from Fig. 4(a), which shows that small intensity fluctuations will introduce a larger reflectivity change of the saturable absorber if the slope is larger. Therefore, the mode-locking build-up time decreases with smaller saturation intensities. However, there is a tradeoff: if the saturation intensity is too small, the laser will start to $Q$ switch. The condition for no $Q$-switching is derived in [4], [9]:

$$
\text { no } Q \text {-switching: }\left|\frac{d R}{d I}\right| I<r \frac{T_{R}}{\tau_{2}} \approx \frac{T_{R}}{\tau_{\text {stim }}} .
$$

where $r$ is the pump parameter that determines how many times the laser is pumped above threshold, $T_{R}$ is the cavity round trip time, and $\tau_{2}$ is the upper state lifetime of the laser. The stimulated lifetime $\tau_{\text {stim }}$ of the upper laser level is given by $\tau_{\text {stim }}=\tau_{2} /(r-1) \approx \tau_{2} / r$ for $r \gg 1$. The small signal gain of the laser is given by $g_{0}=r l$, where $l$ is the total loss coefficient of the laser cavity. From (4), it then follows that $Q$-switching can be more easily suppressed for a small slope $d R / d I$ (i.e., a large saturation intensity), a large $r$ (i.e., a laser that is pumped far above threshold with a large small-signal gain $g_{0}$ or small losses $l$ ), a large cavity round-trip period (i.e., for example a low mode-locked pulse repetition rate). Equation (4) also indicates that solid-state lasers with a large upper state lifetime $\tau_{2}$ will have an increased tendency for self- $Q$-switching instabilities.

The physical interpretation of the $Q$-switching threshold (4) is as follows: The left-hand side of (4) determines the reduction in losses per cavity round-trip due to the bleaching in the saturable absorber. This loss reduction will increase the intensity inside the laser cavity. The right-hand side of (4) determines how much the gain per round-trip saturates, compensating for the reduced losses and keeping the intensity inside the laser cavity constant. If the gain cannot respond fast enough, the intensity continues to increase as the absorber is bleached, leading to self- $Q$-switching instabilities or stable $Q$-switching.

Equations (3) and (4) give an upper and lower bound for the saturation intensity which results in stable $\mathrm{CW}$ mode-locking without self- $Q$-switching. Of course, we can also optimize a saturable absorber for $Q$-switching by selecting a small saturation intensity and a short cavity length, i.e., a short $T_{R}$. This will be discussed in more detail in Section V.

If we use a fast saturable absorber with recovery time much shorter than the cavity round-trip time $\left(T_{A} \ll T_{R}\right)$, then the conditions given by (3) and (4) are typically fulfilled and much shorter pulses can be formed. But now, an additional stability requirement has to be fulfilled to prevent $Q$-switched modelocking (Fig. 1). For this further discussion, we assume that the steady-state pulse duration $\tau_{p}$ is shorter than the recovery time $T_{A}$ of the saturable absorber, i.e., $\tau_{p}<T_{A}$. In this case the saturation [Fig. 4(b)] is determined by the saturation fluence $E_{\text {sat }}$, given by

$$
E_{\mathrm{sat}}=\frac{h \nu}{\sigma_{A}}
$$

and the incident pulse energy density $E_{p}$ on the saturable absorber. The loss reduction per round-trip is now due to bleaching of the saturable absorber by the short pulses, not the CW intensity. This is a much larger effect when $T_{A} \ll T_{R}$. Therefore, in analogy to (4), we can show that the condition to prevent $Q$-switched mode-locking is given by [9]:

no $Q$-switched mode-locking: $\left|\frac{d R}{d E_{p}}\right| E_{p}<r \frac{T_{R}}{\tau_{2}} \approx \frac{T_{R}}{\tau_{\text {stim }}}$

We can easily fulfill this condition by choosing $E_{p} \gg E_{\text {sat }}$ [Fig. 4(b)]. This also optimizes the modulation depth, resulting in reduced pulse duration.

However, there is also an upper limit to $E_{p}$, determined by the onset of multiple pulsing [63]. Given an energy fluence many times the saturation energy fluence $E_{\text {sat }}$, we can see that the reflectivity is strongly saturated and no longer a strong function of the pulse energy. In addition, shorter pulses see a reduced average gain, due to the limited gain bandwidth of the laser. Beyond a certain pulse energy, two pulses with lower power, longer duration, and narrower spectrum will be preferred, since they see a larger increase of the average gain but a smaller increase in the absorption. The threshold for multiple pulsing is lower for shorter pulses, i.e., with spectrums broad compared to the gain bandwidth of the laser. Our experimentally determined rule of thumb for the pulse energy density on the saturable absorber is three to five times the saturation fluence. A more detailed description of multiple pulsing will be given elsewhere. In general, the incident pulse energy density on the saturable absorber can be adjusted by the incident mode area, i.e., how strongly the cavity mode is focused onto the saturable absorber.

Equations (3), (4), and (6) give general criteria for the saturation intensity $I_{\text {sat }}(1)$ and saturation fluence $E_{\text {sat }}(5)$ of the saturable absorber. Normally, the saturation fluence of the absorber material is a given, fixed parameter, and we have to 


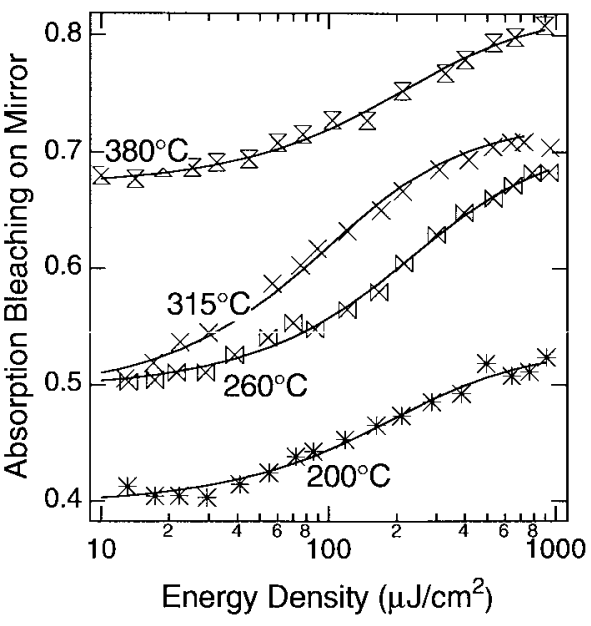

(a)

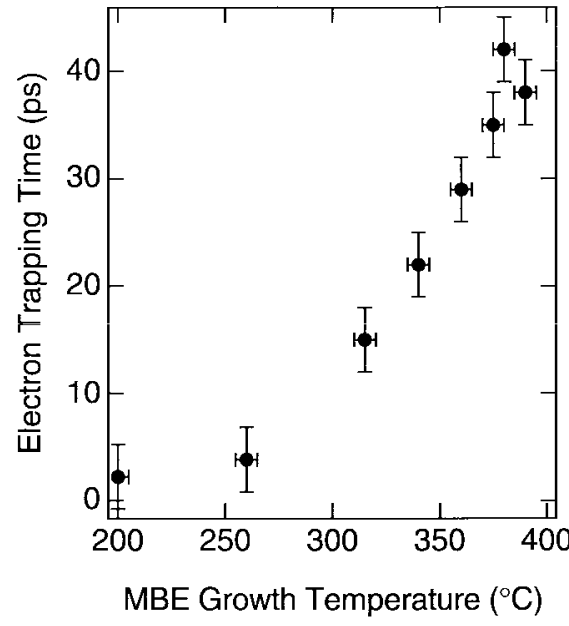

(b)

Fig. 5. Measured absorption bleaching and electron trapping times (i.e., recovery time of saturable absorber) for low-temperature MBE grown InGaAs-GaAs multiple quantum-well absorbers. The MBE growth temperature is the variable parameter used in the nonlinear reflectivity.

adjust the incident mode area to set the incident pulse energy density onto the saturable absorber to fulfill the conditions given by (6) and the multiple pulsing instabilities. Therefore, the only parameter left to adjust for the saturation intensity is the absorber recovery time $T_{A}$ (1). However, if we want to use the absorber as a fast saturable absorber, we have to reduce $T_{A}$. Semiconductor materials are interesting in this regard, because we can adjust $T_{A}$ from the nanosecond to the subpicosecond regime using different growth parameters (Section III-A). In this case, however, it is often necessary to find another parameter with which to adjust $I_{\text {sat }}$ rather than with $T_{A}$. We will show in the next section that this can be obtained by using semiconductor saturable absorbers inside a device structure which allows us to modify the effective absorber cross section $\sigma_{A}$ (1), which is a fixed material parameter. For cases where the cavity design is more restricted and the incident mode area on the saturable absorber is not freely adjustable, modifying the device structure offers an interesting solution for adjusting the effective saturation fluence of the SESAM device to the incident pulse energy density. This is particularly useful for the passively $Q$-switched monolithic ring lasers [64] and microchip lasers [65], [66], discussed in more detail in Section V.

\section{SEMiconductor SATURABle AbSORbER MirRor (SESAM) DESIGN}

\section{A. Material and Device Parameters}

Normally grown semiconductor materials have a carrier recombination time in the nanosecond regime, which tends to drive many solid-state lasers into $Q$-switching instabilities (Section II). In addition, nanosecond recovery times do not provide a fast enough saturable absorber for $\mathrm{CW}$ modelocking. We use low-temperature grown III-V semiconductors [5], [7], [67] which exhibit fast carrier trapping into point defects formed by the excess group- $\mathrm{V}$ atoms incorporated during the LT growth [11], [68], [69]. Fig. 5 shows typical electron trapping times (i.e., absorber recovery times) and the nonlinear absorption bleaching as a function of MBE growth temperature. For growth temperatures as low as $250{ }^{\circ} \mathrm{C}$, we still obtain a good nonlinear modulation of the saturable absorber with recovery times as low as a few picoseconds. The tradeoff here is that the nonsaturable absorber losses for $E_{p} \gg E_{\text {sat }}$ increase with reduced growth temperatures [8]. This tradeoff will ultimately limit the maximum thickness of the absorber material used inside a solid-state laser cavity.

For femtosecond pulse generation, we can benefit from the intraband thermalization processes that occur with time constants from tens to hundreds of femtoseconds, depending on the excitation intensity and energy [70]. A larger femtosecond modulation depth can be obtained for quantum-well structures because of the approximately constant density of states above the bandgap. However, we can strongly reduce the requirements on this fast recovery time if we do not use the semiconductor saturable absorber as a fast saturable absorber, according to Fig. 3(b), but just to start and stabilize soliton mode-locking. In this case, no quantum-well effects are absolutely necessary and, therefore, bulk absorber layers are in most cases sufficient as well. The reduced requirements on the absorber dynamics also allowed us to demonstrate $50-\mathrm{nm}$ tunability of a diode-pumped, soliton-mode-locked Cr:LiSAF laser with a one-quantum-well low-finesse A-FPSA (Fig. 6) [71], [72]. We would not obtain this broad tunability if the excitonic nonlinearities in the SESAM provided the dominant pulse formation process. In addition, in the soliton modelocking regime we can also obtain pulses in the 10-fs range or below, even though the mode-locked spectrum extends beyond the bandgap of the semiconductor saturable absorber, for example [56].

We can further adjust the key parameters of the saturable absorber if we integrate the absorber layer into a device structure. This allows us to modify the effective absorber cross section $\sigma_{A}$ (2) beyond its material value, for example. In addition, we can obtain negative dispersion compensation by using a Gire-Tournois mirror or chirped mirrors. In the following, we will discuss the different device designs in more detail. 

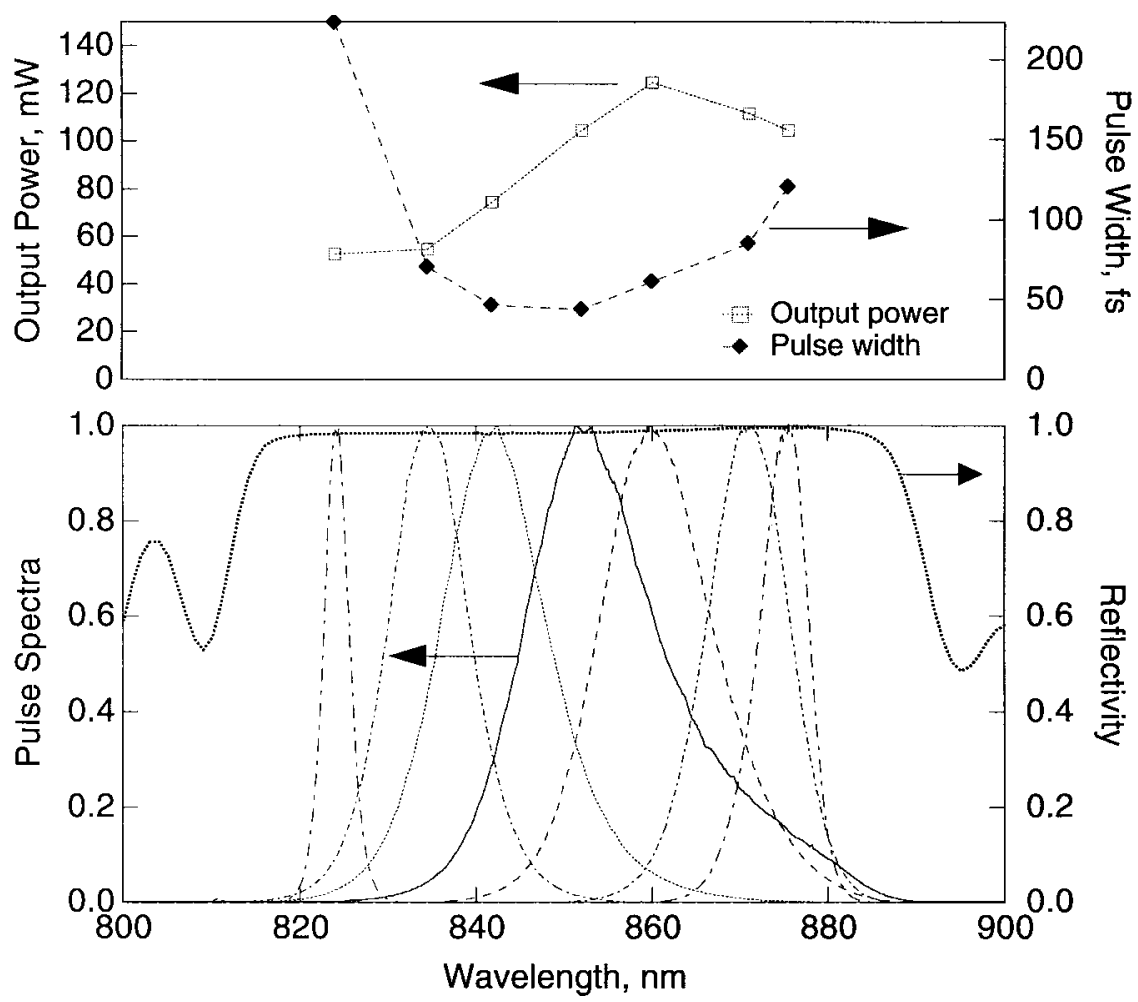

Fig. 6. Tunability of a diode-pumped Cr:LiSAF laser using an intracavity low-finesse A-FPSA. Pulsewidth as short as 45 fs has been achieved. The Tunability of $\approx 50 \mathrm{~nm}$ was limited by the lower AlGaAs-AlAs Bragg mirror of the A-FPSA.

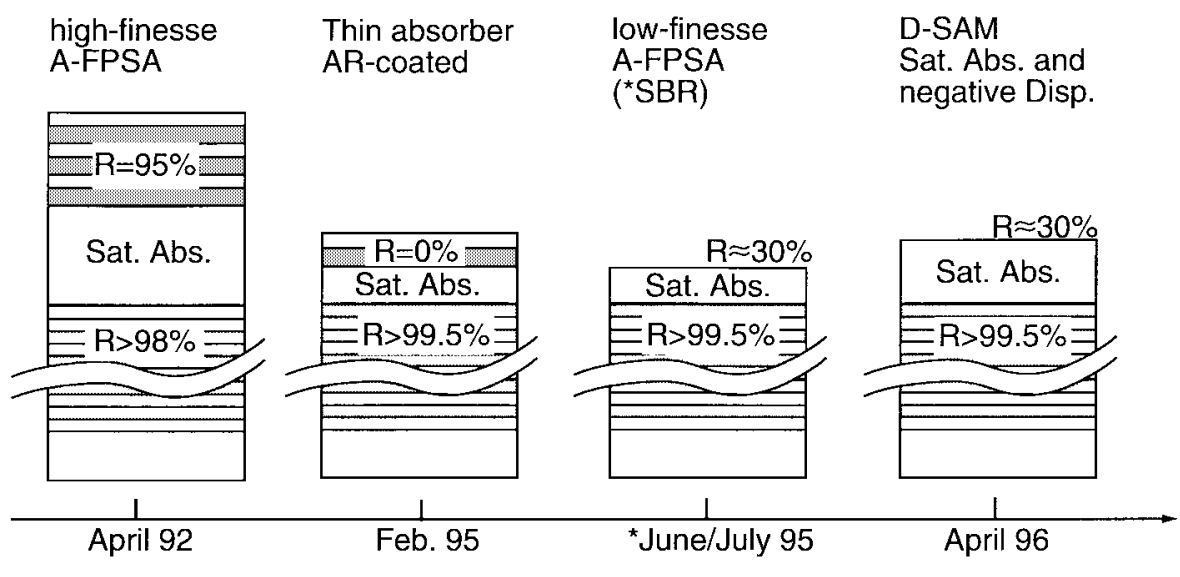

(a)

(b)

(c)

(d)

Fig. 7. Different SESAM devices in historical order. (a) High-finesse A-FPSA. (b) Thin AR-coated SESAM. (c) Low-finesse A-FPSA. (d) D-SAM.

\section{B. Overview of the Different SESAM Designs}

SESAM's offer a distinct range of operating parameters not available with other approaches. We use various designs of SESAM's [73] to achieve many of the desired properties. Fig. 7 shows the different SESAM designs in historical order. The first intracavity SESAM device was the antiresonant Fabry-Perot saturable absorber (A-FPSA) [5], initially used in a design regime with a rather high top reflector, which we call now more specifically the high-finesse A-FPSA. The Fabry-Perot is typically formed by the lower semiconductor Bragg mirror and a dielectric top mirror, with a saturable absorber and possibly transparent spacer layers in between. The thickness of the total absorber and spacer layers are adjusted such that the Fabry-Perot is operated at antiresonance
[(7), Figs. 8 and 9]. Operation at antiresonance results in a device that is broad-band and has minimal group velocity dispersion (Fig. 8). The bandwidth of the A-FPSA is limited by either the free spectral range of the Fabry-Perot or the bandwidth of the mirrors.

The top reflector of the A-FPSA is an adjustable parameter that determines the intensity entering the semiconductor saturable absorber and, therefore, the effective saturation intensity or absorber cross section of the device. We have since demonstrated a more general category of SESAM designs, in one limit, for example, by replacing the top mirror with an AR-coating [Fig. 7(b)] [74]. Using the incident laser mode area as an adjustable parameter, we can adapt the incident pulse energy density to the saturation fluence of the device 


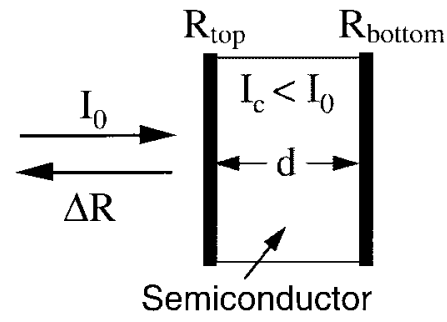

saturable absorber

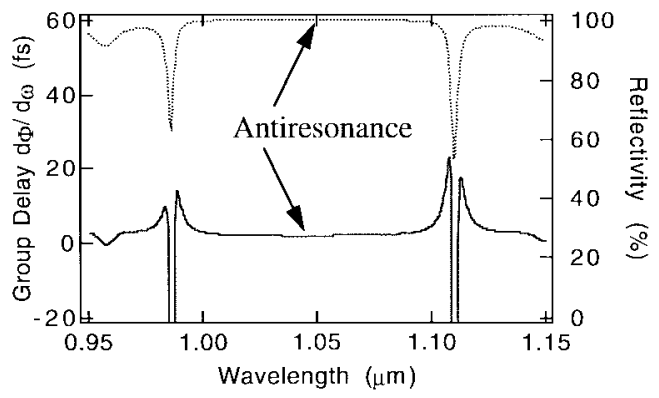

Fig. 8. Basic principle of the A-FPSA concept. With the top reflector, we can control the incident intensity to the saturable absorber section. The thickness of this absorber section is adjusted for antiresonance. The typical reflectivity (dashed line) and group delay (solid line) is shown as a function of wavelength At antiresonance, we have high-broad-band reflection and minimal group delay dispersion.

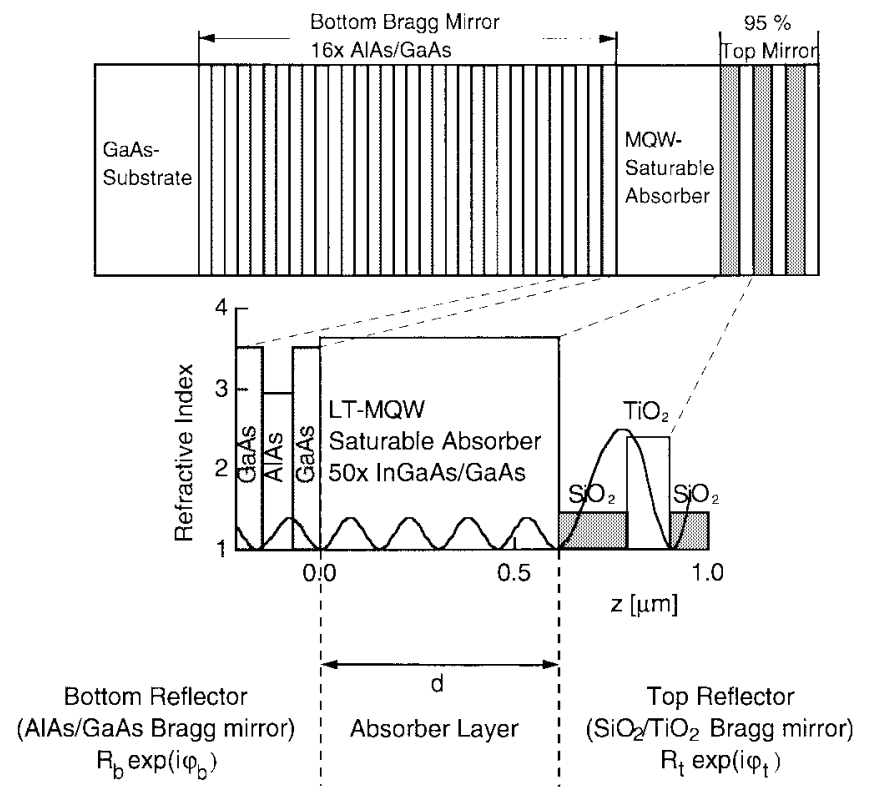

Fig. 9. High-finesse A-FPSA: A specific design for a $\approx 1.05 \mu \mathrm{m}$ center wavelength laser. The enlarged section also shows the calculated standing-wave intensity pattern of an incident electromagnetic wave centered at $1.05 \mu \mathrm{m}$. The Fabry-Perot is formed by the lower AlAs-GaAs Bragg reflector, the absorber layer of thickness $\bar{n} d=4 \cdot \lambda / 2$ and a top $\mathrm{SiO}_{2} / \mathrm{TiO}_{2}$ Bragg reflector, where $\bar{n}$ is the average refractive index of the absorber layer.

(Section II). However, to reduce the nonsaturable insertion loss of the device, we typically have to reduce the thickness of the saturable absorber layer.

A special intermediate design, which we call the low-finesse A-FPSA [Fig. 7(c)] [75]-[77], is achieved with no additional top coating resulting in a top reflector formed by the Fresnel reflection at the semiconductor/air interface, which is typically $\approx 30 \%$.
The dispersive saturable absorber mirror (D-SAM) [78] [Fig. 7(d)] incorporates both dispersion and saturable absorption into a device similar to a low-finesse A-FPSA, but operated close to resonance. The different advantages and tradeoffs of these devices will be discussed below.

\section{High-Finesse A-FPSA}

The high-finesse antiresonant Fabry-Perot saturable absorber (A-FPSA) device [5], [7] (Fig. 9) was the first intracavity saturable absorber that started and sustained stable $\mathrm{CW}$ mode-locking of Nd:YLF and Nd:YAG lasers in 1992. Since then, other solid-state lasers such as Yb:YAG [77], Nd:LSB [79], Nd:YLF, and $\mathrm{Nd}: \mathrm{YVO}_{4}$ at 1.06 and $1.3 \mu \mathrm{m}$ [80] have been passively mode-locked in the picosecond regime with this design. In addition, high-finesse A-FPSA devices have been used to passively $Q$-switch microchip lasers, generating pulses as short as 56 ps [66]. Femtosecond pulse durations $\tau_{p}$ have been generated with Ti:sapphire $\left(\tau_{p}=19 \mathrm{fs}\right)$ [76], Yb:YAG ( $\tau_{p} \approx 500 \mathrm{fs}$ ) [77], diode-pumped $N d:$ glass $\left(\tau_{p}=\right.$ 60-100 fs) [6], [54], [63], and Cr:LiSAF ( $\tau_{p}=45-100 \mathrm{fs}$ ) [52], [72], [81], [82] lasers. In the picosecond regime, the A-FPSA acts as a fast saturable absorber [29], and in the femtosecond regime, mode-locking is typically well-described by the soliton mode-locking model [31]-[33].

Fig. 9 shows a typical high-finesse A-FPSA design for a laser wavelength $\approx 1.05 \mu \mathrm{m}$. The bottom mirror is a Bragg mirror formed by 16 pairs of AlAs-GaAs quarter-wave layers with a complex reflectivity of $R_{b} e^{i \varphi_{b}}$. In this case, the phase shift seen from the absorber layer to the bottom mirror is $\varphi_{b}=\pi$ with a reflectivity of $R_{b} \approx 98 \%$, and to the top mirror $\varphi_{t}=0$ with $R_{t} \approx 96 \%$ [8], [83]. The multiple-quantum-well (MQW) absorber layer has a thickness $d$ chosen such that the antiresonance condition is fulfilled:

$$
\begin{aligned}
\varphi_{r t} & =\varphi_{b}+\varphi_{t}+2 k \bar{n} d \\
& =(2 m+1) \pi
\end{aligned}
$$

where $\varphi_{r t}$ is the round-trip phase inside the Fabry-Perot, $\bar{n}$ is the average refractive index of the absorber layer, $k=2 \pi / \lambda$ is the wavevector, $\lambda$ is the wavelength in vacuum and $m$ is a integer number. From (7), it follows that:

$$
d=m \frac{\lambda}{2 \bar{n}}
$$

From the calculated intensity distribution in Fig. 9, we see that $m=4$.

The $\pi$-phase shift from the lower Bragg reflector in Fig. 9 may seem surprising initially, because the phase shift from the first interface from the MQW absorber layer to GaAs is zero due to the fact that $\bar{n}>n$ (GaAs). However, all the other layers from the AlAs-GaAs Bragg mirror add constructively with a phase shift of $\pi$ at the beginning of the absorber layer. Therefore, this zero-phase reflection is negligible. We also could have chosen to stop the Bragg reflector with the AlAs layer instead of the GaAs layer. However, we typically grow the Bragg reflector during a separate growth run, followed by a regrowth for the rest of the structure. For this reason, we chose to finish the Bragg reflector with the GaAs layer to reduce oxidation effects before the regrowth. 
The saturable absorber layer inside the high-finesse AFPSA (Fig. 9) is typically extended over several periods of the standing wave pattern of the incident electromagnetic wave. This results in about a factor of 2 increase of the saturation fluence and intensity compared to the material value measured without standing-wave effects. We typically measure a saturation fluence of $\approx 60 \mu \mathrm{J} / \mathrm{cm}^{2}$ [8] for an AR-coated (i.e., $R_{t}=0 \%$ ) LT grown InGaAs-GaAs device. With a top reflector the effective saturation fluence is increased as given by (13) and (14) of [8]. For a relatively high top reflector $>95 \%$, the effective saturation fluence is typically increased by about two orders of magnitude.

For a center wavelength around $800 \mathrm{~nm}$, we typically use an AlGaAs-AlAs Bragg mirror with a small enough Ga content to introduce no significant absorption. These mirrors have less reflection bandwidth than the GaAs-AlAs Bragg mirrors because of the lower refractive index difference. However, we have demonstrated pulses as short as 19 fs from Ti:sapphire laser [76] with such a device. In this case, the bandwidth of the mode-locked pulse extends slightly beyond the bandwidth of the lower AlGaAs-AlAs mirror, because the much broader $\mathrm{SiO}_{2} / \mathrm{TiO}_{2}$ Bragg mirror on top reduces bandwidth limiting effects of the lower mirror. Reducing the top mirror reflectivity increases the minimum attainable pulsewidth due to the lower mirror bandwidth.

\section{AR-Coated SESAM}

The other limit of the A-FPSA design is a zero top reflector i.e., an AR-coating (Fig. 7) [74], [76]. Such device designs are shown in Fig. 10 for a Ti:sapphire laser. The thickness of the absorber layer has to be smaller than $d$ to reduce the nonsaturable insertion loss of these intracavity saturable absorber devices. To obtain broad-band performance with no resonance effects, we add transparent AlAs or AlGaAs spacer layers. The limitations of this device include the bandwidth of the lower AlAs-AlGaAs Bragg mirror, and the potentially higher insertion loss compared to the high-finesse A-FPSA.

These AR-coated SESAM's have started and stabilized a soliton mode-locked Ti:sapphire laser achieving pulses as short as 34 fs [for device in Fig. 10(a)] [74] and 13 fs [for device in Fig. 10(b)] [33] with a mode-locking build-up time of only $\approx 3 \mu \mathrm{s}$ and $\approx 200 \mu \mathrm{s}$, respectively. As mentioned before, stable mode-locking was achieved over the full stability regime of the laser cavity. The measured maximum modulation depth $\Delta R$ was $\approx 5 \%$ with a bitemporal impulse response of $230 \mathrm{fs}$ and 5 ps [for the device in Fig. 10(a)] and $\Delta R \approx$ $6 \%$ with a bitemporal impulse response of $60 \mathrm{fs}$ and 700 fs [for device in Fig. 10(b)] measured at the same pulse energy density and pulse duration as inside the Ti:sapphire laser. For the first device [Fig. 10(a)] we were limited in pulsewidth by the bandwidth of the lower AlAs-AlGaAs Bragg mirror [74], which was then replaced by a broad-band silver mirror [Fig. 10(b)]. In addition, the position of the thin saturable absorber layer within the spacer layer was adjusted with respect to the standing wave intensity pattern to adjust the effective saturation fluence, or to partially compensate bandgap-induced wavelength dependence in the latter case.
The AR-coated SESAM device can be viewed as one design limit of the A-FPSA with a $\approx 0 \%$ top reflector [74], [76]. Fig. 10(a) shows a simple AlAs-AlGaAs Bragg reflector with a single-GaAs quantum-well absorber in the last quarterwavelength thick AlAs layer of the Bragg reflector. The additional AR-coating is required to prevent Fabry-Perot effects [74]. The need for this additional AR-coating is maybe not obvious but can be seen in low-intensity reflectivity measurements of this device with and without an AR-coating [Fig. 10(c)]. The reflectivity dip in Fig. 10(c) at $\approx 850 \mathrm{~nm}$ is due to the absorption in the GaAs quantum-well and corresponds to a Fabry-Perot resonance. This strong wavelength dependent reflectivity prevents short pulse generation and pushes the lasing wavelength of the Ti:sapphire laser to the high-reflectivity of the device at shorter wavelength at the edge of the Bragg mirror [74]. The Fabry-Perot in Fig. 10(a) that explains this resonance dip is formed by the lower part of the AlAs-AlGaAs Bragg reflector, the transparent AlAs layer with the GaAs absorber quantum-well layer of total thickness $\bar{n} d=\lambda / 4$ and the Fresnel reflection of the last semiconductor/air interface (without AR-coating), where $\bar{n}$ is the average refractive index of the last AlAs and GaAs layer. This Fabry-Perot is at resonance because the round-trip phase shift $\varphi_{r t}$ is according to (7):

$$
\begin{aligned}
\varphi_{r t} & =\varphi_{b}+\varphi_{t}+2 k \bar{n} d \\
& =\pi+0+\pi \\
& =2 \pi .
\end{aligned}
$$

A $\varphi_{r t}$ of $2 \pi$ allows for constructive interference and therefore fulfills the resonance condition of the Fabry-Perot. No ARcoating would be required if the AlAs-AlGaAs Bragg reflector in Fig. 10(a) would end with the quarter-wavelength-thick AlGaAs layer that then incorporates the GaAs quantum-well. In this case, the phase shift of the lower part of the Bragg mirror is $\varphi_{b}=0$ instead of $\pi$ (9) and, therefore, $\varphi_{r t}=$ $\pi$, the condition for antiresonance (7). This design would correspond to a specific low-finesse A-FPSA or also referred to as the saturable Bragg reflector [see next Section III-E and Fig. 11(b)]. An additional AR-coating, however, increases the modulation depth of this device and acts as a passivation layer for the semiconductor surface that can improve long-term reliability of this SESAM device.

\section{E. Low-Finesse A-FPSA}

The two design limits of the A-FPSA are the high-finesse A-FPSA [Fig. 7(a)] with a relatively high top reflector (i.e.,>95\%) and the AR-coated SESAM [Fig. 7(b)] with no top reflection (i.e., $R_{t} \approx 0 \%$ ) [74]. Using the incident laser mode area as an adjustable parameter, the incident pulse energy density $E_{p}$ can be adapted to the saturation fluence $E_{\text {sat }}$ of both SESAM's for stable mode-locking by choosing $E_{p}$ a few times $E_{\text {sat }}$ (see Section II) [76]. A specific intermediate design is the low-finesse A-FPSA [75]-[77], where the top reflector is formed by the $\approx 30 \%$ Fresnelreflection of the semiconductor/air interface [Fig. 7(c) and Fig. 11]. Reducing the top reflector typically requires a thinner 


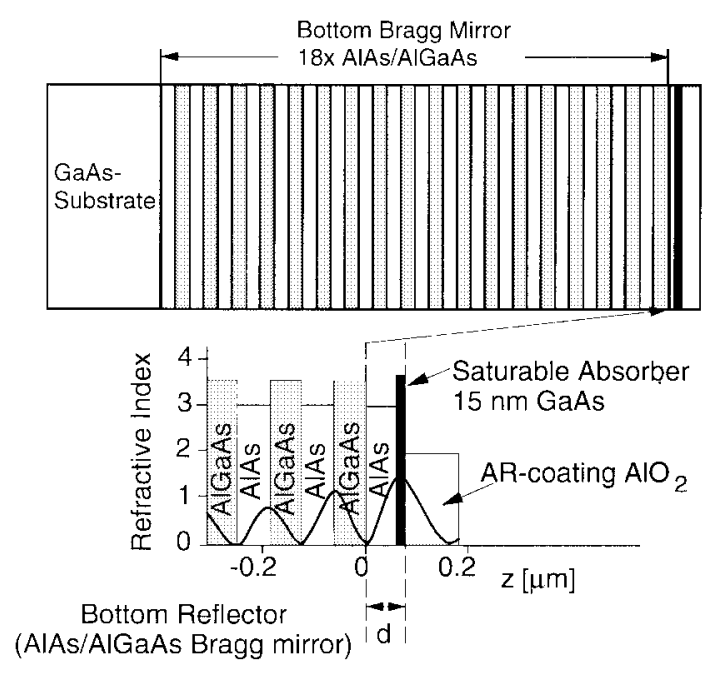

(a)

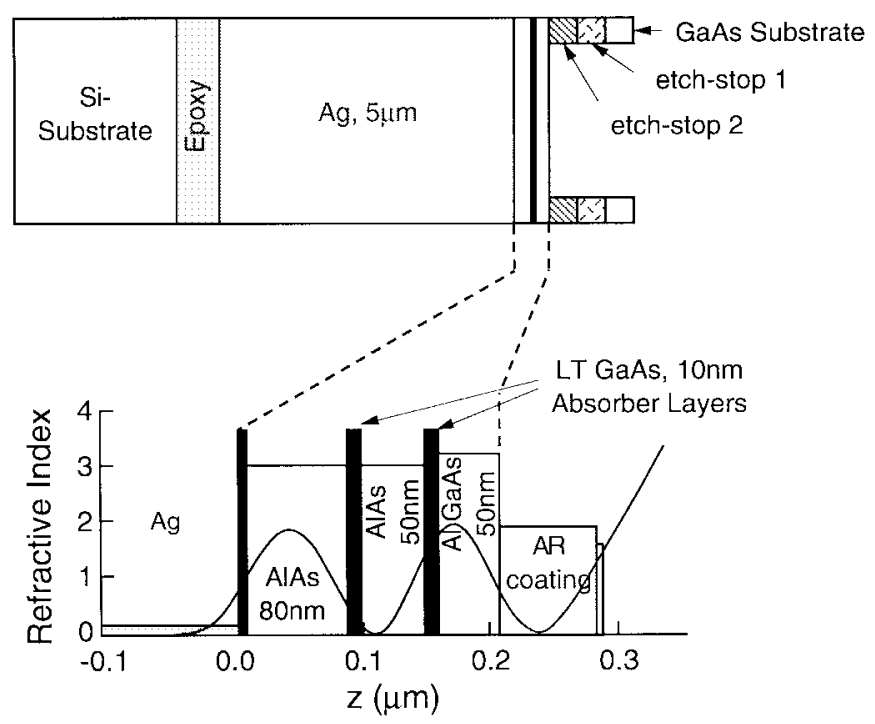

(b)

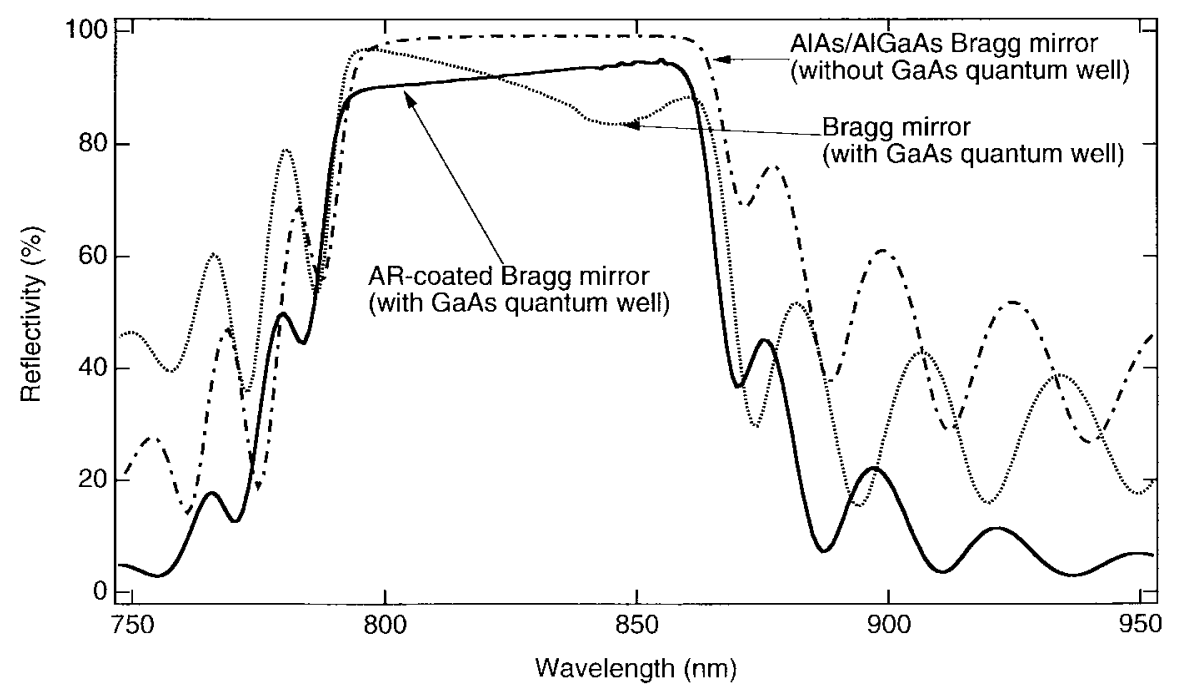

(c)

Fig. 10. AR-coated SESAM: Two specific designs for a $\approx 800-\mathrm{nm}$ center wavelength laser such as Ti:sapphire or Cr:LiSAF. (a) The basic structure is a AlAs-AlGaAs Bragg reflector with a single GaAs quantum well as the saturable absorber. The additional AR-coating is required to prevent Fabry-Perot effects [see Fig. 10(c)]. The bandwidth is limited to $\approx 30$ fs pulses by the lower AlGaAs-AlAs Bragg mirror. (b) Broad bandwidth for sub-10-fs pulse generation is obtained by replacing the Bragg mirror with a silver mirror. This device, however, requires post-growth etching to remove the GaAs substrate and etch-stop layers from the absorber-spacer layer. (c) Low-intensity reflectivity of the AlAs-AlGaAs Bragg reflector without a GaAs quantum-well absorber, with a GaAs absorber and with both a GaAs absorber and the AR-coating [according to Fig. 10(a)].

saturable absorber and a higher bottom reflector to minimize nonsaturable insertion loss.

Fig. 11(a) shows a specific design for a wavelength $\approx 1.05$ $\mu \mathrm{m}$. Similar to the high-finesse A-FPSA (Fig. 9), the bottom mirror is a Bragg mirror formed by 25 pairs of AlAs-GaAs quarter-wave layers with a complex reflectivity of $R_{b} e^{i \varphi_{b}}$ with $R_{b}>99 \%$. The thickness $d$ of the spacer and absorber layers are adjusted for antiresonance (7), with $\varphi_{b}=\pi[83]$ and $\varphi_{t}=$ 0 which gives a minimal thickness of $\lambda / 2 \bar{n}$ for $m=1$ (8). The residual reflection from the different spacer and absorber layers is negligible in comparison to the accumulated reflection from the lower multilayer Bragg reflector and the semiconductorair interface. This is also confirmed by the calculated standing wave intensity pattern shown in Fig. 11(a). Because there is no special surface passivation layer, it is advantageous for a higher damage threshold to have a node of the standing wave intensity pattern at the surface of the device [78].

Independently, a similar low-finesse A-FPSA device for a center wavelength $\approx 860 \mathrm{~nm}$ [Fig. 11(b)] was introduced, termed the saturable Bragg reflector (SBR) [75]. This device is very similar to the previously introduced AR-coated SESAM device [74] shown in Fig. 10(a). In this case, however, no AR-coating is required on the AlAs-AlGaAs Bragg reflector. This can be explained with the A-FPSA design concept: We can also describe this SBR device as a low-finesse A-FPSA [Fig. 11(b)], consisting of a lower AlAs/AlGaAs Bragg mirror plus a quarter-wave thick Fabry-Perot cavity at anti-resonance (the lowest possible order and thickness). The thickness $d$ of the spacer/absorber layer is adjusted for antiresonance (7), with $\varphi_{b}=0$ [83] and $\varphi_{t}=0$, which gives a minimal 


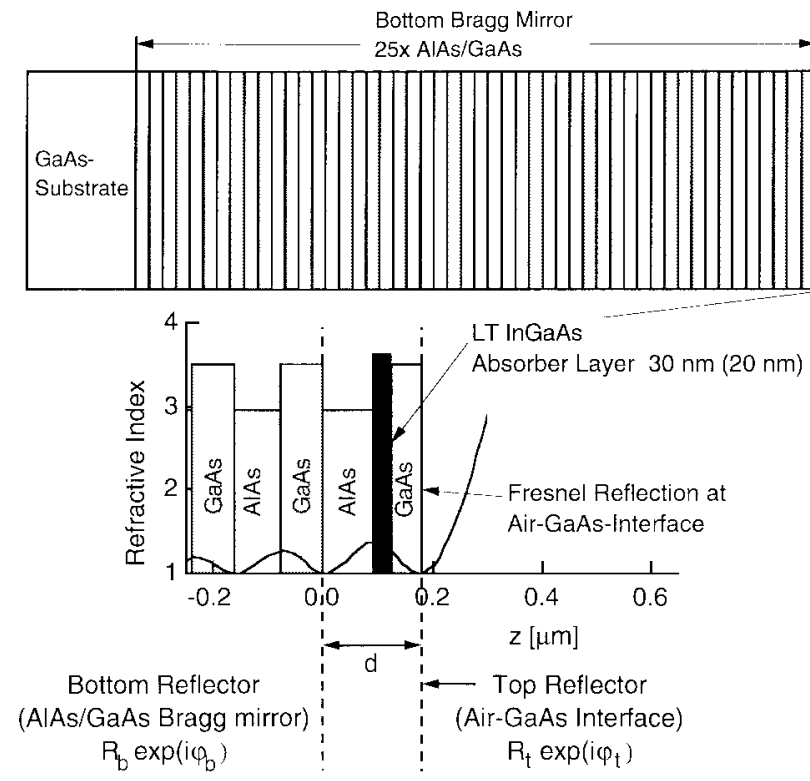

(a)
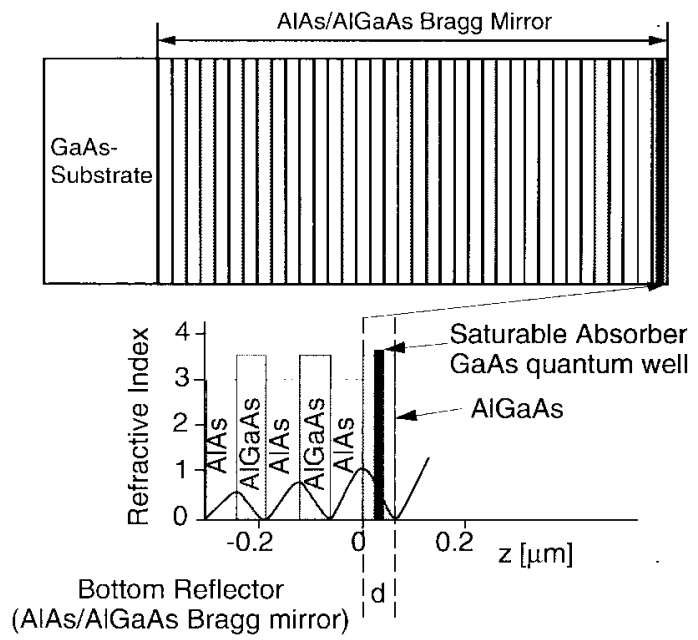

(b)

Fig. 11. Low-finesse A-FPSA: (a) A specific design for a $\approx 1.05 \mu \mathrm{m}$ center wavelength laser. In contrast to the high-finesse A-FPSA in Fig. 9, here the Fabry-Perot is formed by the lower AlAs-GaAs Bragg reflector, the absorber-spacer layer of thickness $\bar{n} d=\lambda / 2$ and the Fresnel reflection from the semiconductor-air interface. Again, the thickness $d$ is adjusted for antiresonance (7). (b) Another specific design for a $\approx 860 \mathrm{~nm}$ center wavelength laser. This device was also called saturable Bragg reflector (SBR) [75] and corresponds to a low-finesse A-FPSA, where the Fabry-Perot is formed by the lower AlAs-AlGaAs Bragg reflector, the absorber-spacer layer of thickness $\bar{n} d=\lambda / 4$ and the Fresnel reflection from the semiconductor-air interface. Again, the thickness $d$ is adjusted for antiresonance (7).

thickness of $\lambda / 4 \bar{n}$ for $m=0$ (8). A saturable absorber is then located inside this Fabry-Perot. With this device pulses as short as 90 fs have been reported with a Ti:sapphire laser [84], which are significantly longer than the 34 fs pulses obtained with the similar AR-coated SESAM device [Fig. 10(a)]. This is most likely due to the lower modulation depth of this device. It is important to realize that the Bragg reflector does not play a key role in its operation and does not actually saturate. For example, the Bragg reflector can be replaced by a metal reflector [Fig. 12(a)] as discussed above to obtain larger bandwidth.

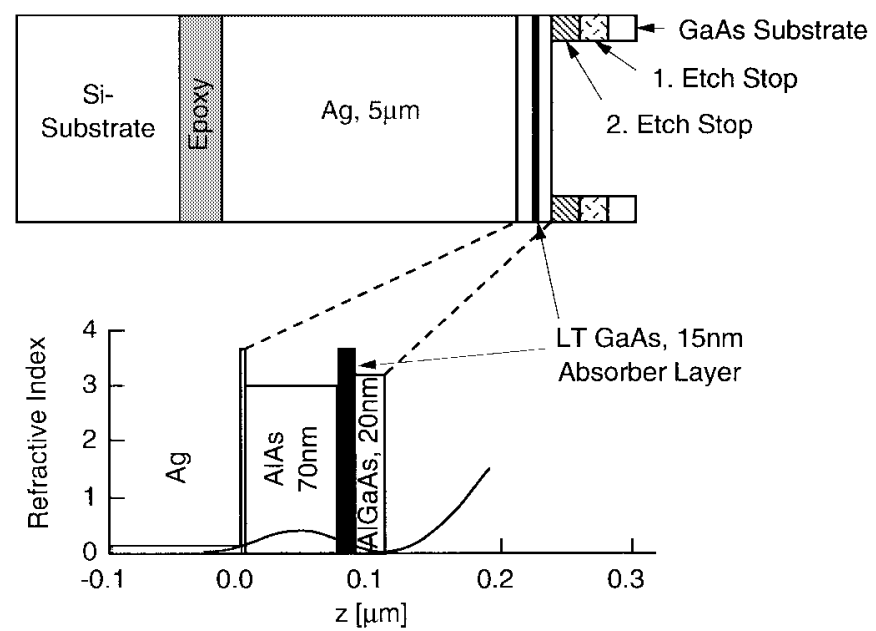

(a)

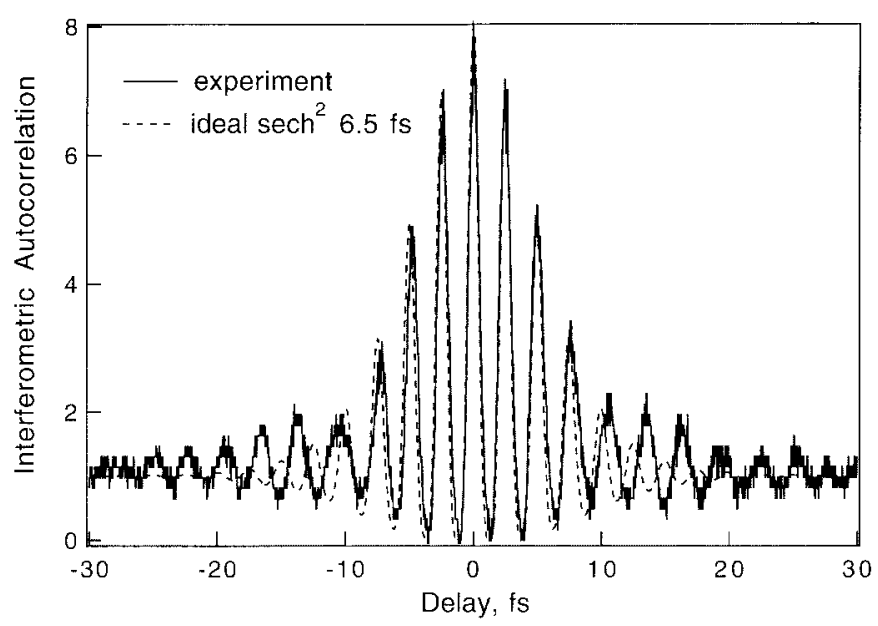

(b)

Fig. 12. Shortest pulses achieved with an intracavity SESAM device: (a) broad-band low-finesse A-FPSA device used for sub-10-fs pulse generation and (b) interferometric autocorrelation of 6.5-fs pulses from a Ti:sapphire laser. The shortest pulses ever produced directly out of a laser without any further pulse compression techniques.

An earlier version of a nonlinear or saturable AlAs-AlGaAs Bragg reflector design was introduced by Kim et al. in 1989 [85]. In this case, the nonlinear Bragg reflector operates on saturable absorption due to band filling in the narrower bandgap material of the Bragg reflector. This results in a distributed absorption over many layers. This device, however, would introduce too much loss inside a solid-state laser. Therefore, only one or a few thin absorbing sections inside the quarter-wave layers of the Bragg reflector are required. The effective saturation fluence of the device can then be varied by changing the position of the buried absorber section within the Bragg reflector or simply within the last quarterwave layer of the Bragg reflector, taking into account that a very thin absorber layer at the node of a standing wave does not introduce any absorption.

The limitations of these SESAM devices include the bandwidth of the lower Bragg mirror, and potentially higher insertion loss than in the high-finesse A-FPSA. Pulses as short as $19 \mathrm{fs}$ have been generated with the high-finesse A-FPSA 
compared to $34 \mathrm{fs}$ with the low-finesse A-FPSA using the same lower Bragg mirror, for example [76]. Replacing the lower Bragg mirror with a broad-band silver mirror [Fig. 12(a)] resulted in self-starting 10-fs pulses [56] and more recently pulses as short as 6.5 fs [44] [Fig. 12(b)] with a KLM-assisted Ti:sapphire.

\section{F. D-SAM}

Many applications require more compact and simpler femtosecond sources with a minimum number of components. Intracavity prism pairs for dispersion compensation typically limit the minimum size of femtosecond laser resonators. Alternative approaches have been investigated for replacing the prism pairs by special cavity resonator designs incorporating more compact angular dispersive element. For example, a prismatic output coupler [86], or similarly only one prism [87], has supported pulses as short as $110 \mathrm{fs}$ with a Ti:sapphire laser, or 200-fs pulses with a diode-pumped $\mathrm{Nd}$ :glass laser, respectively. In both cases, the basic idea can be traced back to the prism dispersion compensation technique [88]. Chirped mirrors [42], [89], [90], mentioned earlier, are compact dispersion compensation elements, but typically require multiple reflections to achieve sufficient dispersion compensation. A Gires-Tournois mirror [91] is also a compact dispersion compensation technique, but has a tradeoff in terms of bandwidth and tunability.

Recently, we combined both saturable absorption and dispersion compensation in a semiconductor Gires-Tournoislike structure, called a dispersion-compensating saturable absorber mirror (D-SAM) [Fig. 7(d)] [78]. By replacing one end mirror of a diode-pumped Cr:LiSAF laser with this device, we achieved 160-fs pulses without further dispersion compensation or special cavity design. This is the first time that both saturable absorption and dispersion compensation have been combined within one integrated device. The DSAM, in contrast to the A-FPSA, is operated close to the Fabry-Perot resonance, which tends to limit the available bandwidth of the device. In the future, chirped mirror designs that incorporate saturable absorber layers could also potentially provide both saturable absorption and negative dispersion, but with potentially more bandwidth.

\section{G. A-FPMod}

We do not have to rely only on passive saturable absorption with semiconductors. Multiple-quantum-well (MQW) modulators based on the quantum-confined Stark effect [92]-[94] are promising as active modulation devices for solid-state lasers, sharing the same advantages of passive SESAM's: they are compact, inexpensive, fast, and can cover a wide wavelength range from the visible to the infrared. In addition, they only require a few volts of drive voltage or several hundred milliwatts of RF power. In general, however, semiconductor MQW modulators would normally introduce excessive insertion losses inside a solid state laser cavity and would also saturate at relatively low intensities [95], [96]. We extended the antiresonant Fabry-Perot principle by integrating an active MQW modulator inside a Fabry-Perot structure, which we called antiresonant Fabry-Perot Modulator (A-FPMod) [97]. We then actively mode-locked a diode-pumped Nd:YLF laser.

One advantage of quantum-well modulators compared to other modulators such as acoustooptic modulators or phase modulators is that they also can act as saturable absorbers leading to passive mode-locking with much shorter pulses. Combining the effects of saturable absorption and absorption modulation within one single device, we have demonstrated the possibility to synchronize passively mode-locked pulses to an external RF signal [97]. At higher output powers we were limited by the increased saturation of the active modulator.

\section{An All-Solid-State Ultrafast Laser \\ TECHNOLOGY: PASSIVElY MODELOCKED DIODE-PUMPED SOLID-STATE LASERS}

In the last few years, we have seen first demonstrations of potentially practical ultrafast solid-state lasers. Our approach for practical or "real-world" ultrafast lasers is as follows: For simplicity, reliability, and robustness, we only consider diode-pumped solid-state lasers with passive mode-locking or $Q$-switching techniques, where we use SESAM's to provide efficient pulse formation and stabilization. In addition, we do not want to rely on critical cavity alignment and therefore use fast saturable absorber mode-locking in the picosecond regime and soliton mode-locking in the femtosecond regime. The general goal is to develop a compact, reliable, easy-to-use, "hands-off" all-solid-state ultrafast laser technology.

\section{A. Cr:LiSAF}

Diode-pumped broad-band lasers are of special interest for number of practical applications. Ti:sapphire is probably the best known of the ultrafast lasers, but must be pumped in the green spectral region, were no high-power diode lasers yet exist. However, the fairly newly developed Cr:LiSAF family of crystals (Cr:LiSAF [98], Cr:LiCAF [99], Cr:LiSCAF [100], and Cr:LiSGAF [101]) have fluorescence linewidths similar to Ti:sapphire and can be pumped at wavelengths near $670 \mathrm{~nm}$ where commercial high-brightness high-power (i.e., $<0.5 \mathrm{~W}$ ) diode arrays are available. However, these crystals have a stronger tendency for upperstate lifetime quenching [102] and suffer from lower thermal conductivity, resulting in nonideal performance (limited average power) at relative low pump powers.

The output power of a diode-pumped Cr:LiSAF laser was initially limited to $<10 \mathrm{~mW}$ [103]-[105]. French et al. used an MQW SESAM inside a coupled cavity for RPM [103], [104] and inside the main cavity producing pulses as short as $220 \mathrm{fs}$ in 1994 [105]. However, their device introduced too much fixed losses. Shortly afterwards, we demonstrated [106], [107] significantly higher average output power of 140$\mathrm{mW} \mathrm{CW}$ and $50-\mathrm{mW}$ mode-locked with pulses as short as 98 fs using two 0.4-W high-brightness diode arrays, improved pump mode matching, and a low-loss, high-finesse A-FPSA . Within a year, we improved the pulse duration to $45 \mathrm{fs}$ with a mode-locked average output power of $60 \mathrm{~mW}$ and later then $80 \mathrm{~mW}$ [71], [72], [81] (Fig. 13). Briefly afterwards, Tsuda et al. [75] used a low-finesse A-FPSA design (they 


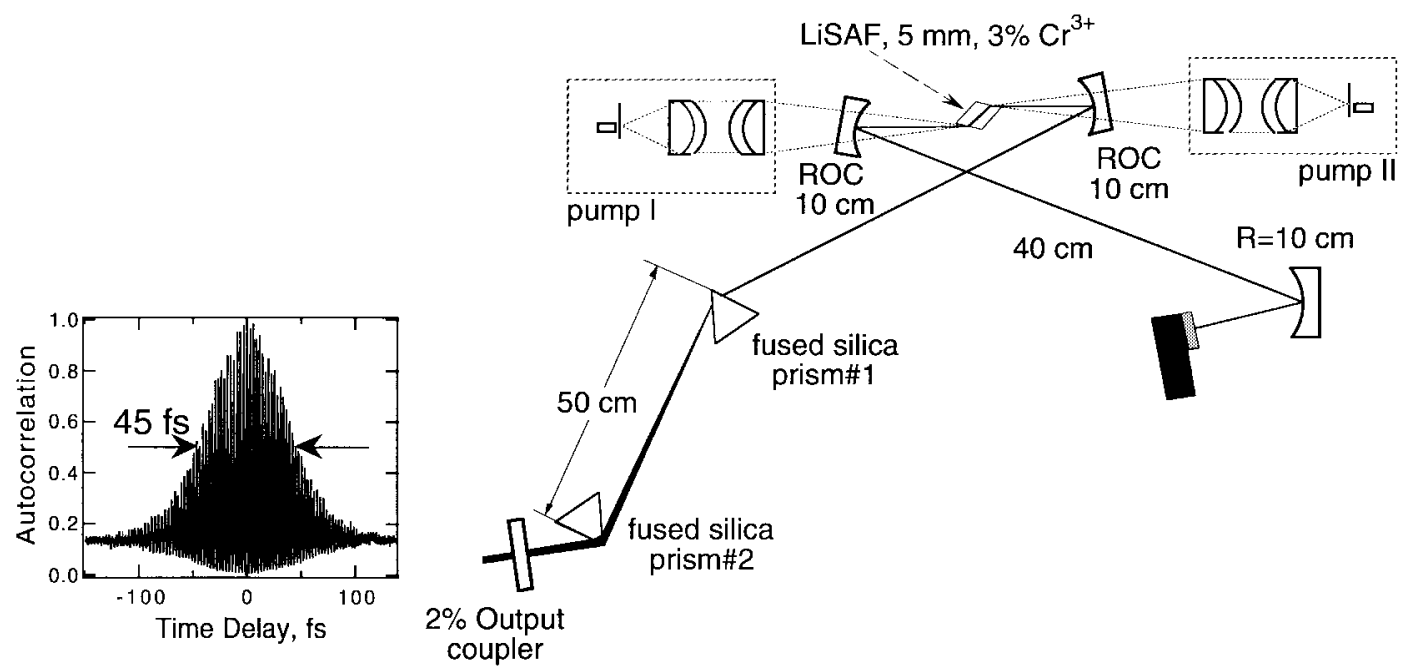

Fig. 13. Diode-pumped Cr:LiSAF laser cavity setup that generated pulses as short as 45 fs with soliton mode-locking.

termed it "SBR," see Section III-E) inside a diode-pumped Cr:LiSAF laser and demonstrated 100-fs pulses with 11-mW average output power. Recently, they improved this result with a MOPA diode laser, which provides a near-diffraction-limited 0.5 -W pump, achieving 70 -fs pulses with $100-\mathrm{mW}$ output power [108].

Significant efforts by many groups around the world are directed toward shorter pulses and more output power. Presently, the shortest pulses of $\approx 20$ fs have been obtained with KLM Cr:LiSAF [109], [110] with an average output power in the regime of $1 \mathrm{~mW}$. Higher output power has been achieved only at the expense of longer pulses. For example, 40-fs pulses with an average output power of $70 \mathrm{~mW}$ have been recently obtained [110] in a KLM Cr:LiSAF system.

The limited average output power of femtosecond diodepumped Cr:LiSAF lasers is their main drawback in comparison to Ti:sapphire lasers. Novel diode pumping techniques can address this problem, and we have achieved $400 \mathrm{~mW}$ [111] and more recently as much as $1.4-\mathrm{W} \mathrm{CW}$ output power from a diode-pumped Cr:LiSAF laser (Fig. 14) [82], [112]. We have passively mode-locked this laser with a low-finesse A-FPSA [82] and obtained pulses as short as 50 fs with an average output power of $340 \mathrm{~mW}$. Higher average output power of 500 $\mathrm{mW}$ was achieved with 110 -fs pulses. These are the highest average power levels ever achieved to date with femtosecond diode-pumped solid-state lasers. Furthermore, the results show no fundamental limitations for further improvements in both shorter pulses and higher output powers. In contrast to Kerrlens mode-locking, soliton mode-locking with SESAM's has the advantage that the laser cavity mode is decoupled from mode-locking dynamics. This is important in our case, because the cavity design can be more easily optimized for high-power without having to take Kerr-lensing effects into account as well.

\section{B. Nd:glass}

Diode-pumped femtosecond Nd:glass lasers offer a costeffective and compact alternative to Ti:sapphire lasers operated near $1.06 \mu \mathrm{m}$, with applications such as seeding high-power

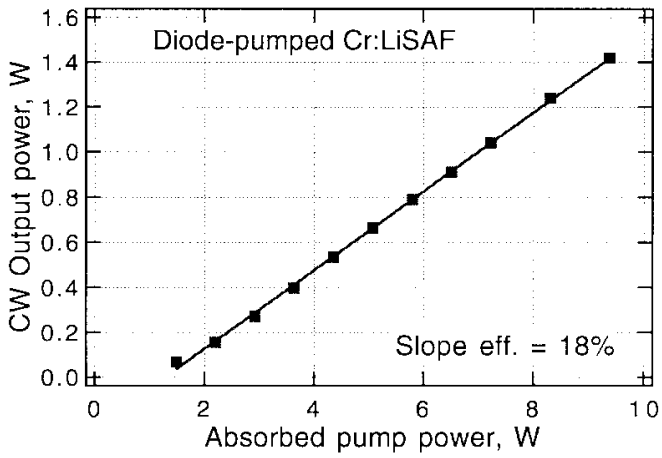

Fig. 14. 1.4-W CW output power from a diode-pumped Cr:LiSAF laser.

amplifiers, pumping femtosecond optical parametric oscillators, and ultrafast spectroscopy. The absorption band of $\mathrm{Nd}$ :glasses at $\approx 800 \mathrm{~nm}$ allows for diode pumping [113], resulting in a compact, wall-plug driven setup, which does not require water cooling. Typical Nd:glasses have a fluorescence bandwidth of 20-30 nm FWHM, supporting sub-100-fs pulse generation at a wavelength of $\approx 1.06 \mu \mathrm{m}$. Initially, the shortest pulses from a (bulk) Nd:phosphate laser [114], $88 \mathrm{fs}$, were produced by additive pulse mode-locking (APM). High-finesse A-FPSA's in various Nd:glass lasers [6], [54] have supported pulses as short as $130 \mathrm{fs}$ for diode pumping and 90 fs for Ti:sapphire pumping.

Recently, we have demonstrated 60-fs pulses [Fig. 15(a)] with an average output power of $\approx 80 \mathrm{~mW}$ from an optimized diode-pumped Nd:glass (Nd:fluorophoshate, LG-810, 3\% Nd) laser using a low-finesse A-FPSA with a larger modulation depth of $\approx 1 \%$, but at the expense of higher intracavity loss (at low intensities) of 2\% [63]. We measured a bitemporal impulse response of the A-FPSA with a fast recovery time of $200 \mathrm{fs}$ and a slow recovery time of 25 ps. The pulse energy density incident on the saturable absorber was typically a few times above the saturation fluence of $\approx 100 \mu \mathrm{J} / \mathrm{cm}^{2}$, limited by the onset of multiple pulsing instabilities. The laser cavity is similar to the diode-pumped Cr:LiSAF laser shown in Fig. 13. The mode-locked spectrum of the $60-\mathrm{fs}$ pulses is $21.6-\mathrm{nm}$ wide 


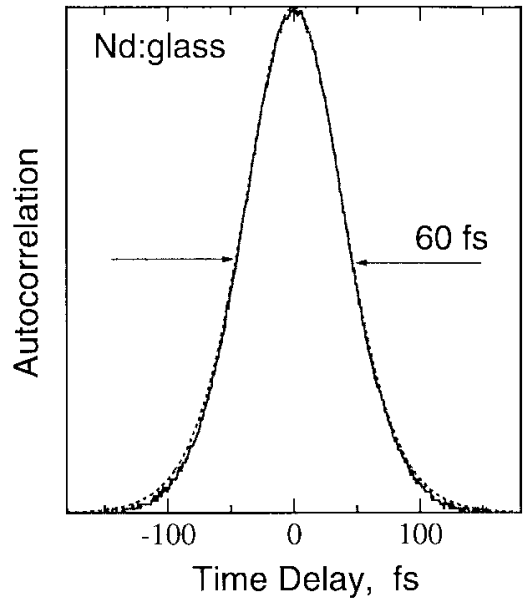

(a)

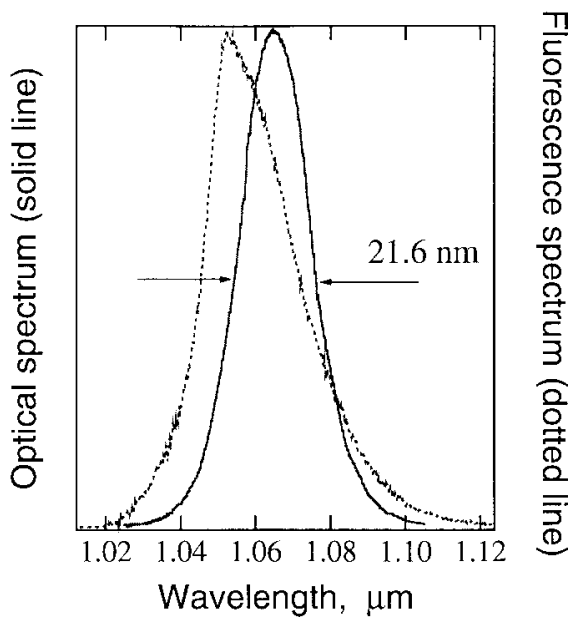

(b)

Fig. 15. Soliton mode-locked Nd:glass (fluorophosphate LG 810) laser using a low-finesse A-FPSA. (a) noncollinear autocorrelation, (b) optical spectrum (solid line) and fluorescence spectrum of $\mathrm{Nd}$ :glass laser (dotted line).

(FWHM) and spreads over most of the available Nd:glass fluorescence bandwidth [Fig. 15(b)]. The mode-locking is selfstarting and is well-discribed by our soliton mode-locking model.

\section{C. $Y b: Y A G$}

Yb:YAG is interesting as a high-power diode-pumped laser source due to its small quantum defect, resulting in a potentially very efficient laser with low thermal loading, and its wide absorption band at $940 \mathrm{~nm}$ [115], [116]. Additionally, Yb:YAG has a broad emission spectrum supporting tunability [117], [118] and femtosecond pulse generation in the few 100fs regime. Using a low-finesse A-FPSA, we have demonstrated a passively mode-locked Yb:YAG laser, generating stable and self-starting pulses as short as 540 fs with typical average output powers of $150 \mathrm{~mW}$ [77]. Yb:YAG has never been passively mode-locked before, because the upper state lifetime of the laser is relatively long, $\approx 1 \mathrm{~ms}$. Previously, only active mode-locking in Yb:YAG has been demonstrated with a pulse duration of 80 ps [119].

Recently, we further optimized the pulse duration with a higher modulation depth of $\approx 1 \%$ for the low-finesse A-FPSA

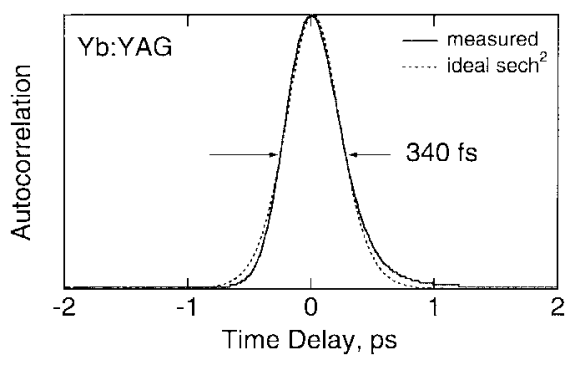

Fig. 16. Soliton mode-locked Yb:YAG laser using a low-finesse A-FPSA.

and obtained pulses as short as 340 fs (Fig. 16) at a center wavelength of $1.033 \mu \mathrm{m}$ and with a spectral width of 3.2-nm FWHM. The measured bitemporal impulse response showed a fast component of 460 fs and a slow recovery time of $\approx 7$ ps. The cavity setup is otherwise the same as in [77].

\section{Nd:YAG, YLF, LSB, and $Y V \mathrm{O}_{4}$}

In the picosecond pulse regime, we use SESAM's as fast saturable absorbers, controlling the recovery time by lowtemperature MBE grown semiconductors (Fig. 5). A more detailed review with regard to $\mathrm{Nd}$ :YAG and $\mathrm{Nd}$ :YLF using a high-finesse A-FPSA is given in [7]. The results for the various laser crystals are summarized in Table I. With picosecond lasers, we achieve significantly shorter pulses if we use the gain material at the end of a linear cavity. This "gainat-the-end" leads to enhanced special hole burning (SHB) that effectively inhomogenously broadens the gain bandwidth, flattening the saturated gain profile and allowing for a larger lasing bandwidth [120]-[122], resulting in shorter pulses. However, we typically observe a time-bandwidth product that is between $1.2-2$ times as large as for ideal transform-limited Gaussian or sech ${ }^{2}$ pulse. This large time-bandwidth product is mainly due to the flat gain produced by SHB, which produces a non-Gaussian pulse shape, and is not due to a chirp on the pulse that could be compensated externally [122].

We have also extended the designs of SESAM's to longer wavelength such as $1.3 \mu \mathrm{m}$ [80] and $1.5 \mu \mathrm{m}$ [123]. To benefit from the good quality of AlAs-GaAs Bragg mirrors, we chose to grow the 1.3- $\mu \mathrm{m}$ saturable absorber layer on a GaAs substrate. To achieve saturable absorption at $1.3 \mu \mathrm{m}$, however, the indium concentration in the InGaAs absorber material must be increased to approximately $40 \%$, which results in a significant lattice mismatch to the GaAs substrate. This lattice mismatch reduces the surface quality, resulting in higher insertion losses, and reduced laser power. In addition, these devices exhibit more bulk defects than a $1-\mu \mathrm{m}$ SESAM, also increasing insertion loss. This is even more enhanced for the $1.5-\mu \mathrm{m}$ saturable absorber. In addition to the short picosecond recovery time of the saturable absorber, the low-temperature MBE growth partially relieves the lattice mismatch, resulting in improved optical quality of the absorber layer.

\section{PASSIVEly $Q$-SWITCHED MicROCHIP LASERS}

In a $Q$-switched laser, the pulse duration generally decreases with shorter cavities and with higher pump power (increased small-signal gain). For solid-state lasers, typically 
TABLE I

Summary of Passively Modelocked Diode-Pumped Nd-doped Laser Materials for Picosecond Pulse Generation Using Intracavity SeSam Devices. The Minimal Pulse Durations are Given for Diode-Pumped Lasers. The Pulsewidths in Parenthesis were Achieved with Ti:sapphire Laser Pumping

\begin{tabular}{l|l|l|l}
\hline Laser material & Center wavelength & \multicolumn{1}{|c}{ Pulse width } & \multicolumn{1}{c}{$\begin{array}{c}\text { Average output } \\
\text { power }\end{array}$} \\
\hline $\mathrm{Nd}: \mathrm{Y} \Lambda \mathrm{G}$ & $1.064 \mu \mathrm{m}$ & $8.3 \mathrm{ps}(6.8 \mathrm{ps})[5,7,124]$ & $\approx 300 \mathrm{~mW}$ \\
\hline $\mathrm{Nd}: \mathrm{YLF}$ & $1.047 \mu \mathrm{m}$ & $5 \mathrm{ps}(2.8 \mathrm{ps})[5,7,124]$ & $\approx 300 \mathrm{~mW}$ \\
\hline & $1.314 \mu \mathrm{m}$ & $5.7 \mathrm{ps}[80]$ & $130 \mathrm{~mW}$ \\
\hline $\mathrm{Nd}: \mathrm{LSB}$ & $1.062 \mu \mathrm{m}$ & $2.8 \mathrm{ps}(1.6 \mathrm{ps})[79]$ & $400 \mathrm{~mW}$ \\
\hline $\mathrm{Nd}: \mathrm{YVO}_{4}$ & $1.064 \mu \mathrm{m}$ & $<10 \mathrm{ps}[125]$ & $>2 \mathrm{~W}$ \\
\hline & $1.34 \mu \mathrm{m}$ & $4.6 \mathrm{ps}[80]$ & $50 \mathrm{~mW}$ \\
\hline
\end{tabular}

$Q$-switched pulsewidths range from nanoseconds to microseconds. Pulsewidths less than a nanosecond ("ultrafast" by $Q$-switching standards) have recently been achieved by using diode-pumping and very short cavity lengths. Extremely short cavity length, typically less than $1 \mathrm{~mm}$, allows for singlefrequency $Q$-switched operation with pulsewidths well below a nanosecond. These $Q$-switched "microchip" lasers are compact and simple solid-state lasers which can provide high peak power with a diffraction limited output beam. Pulse durations of $337 \mathrm{ps}$ and more recently 218 ps have been demonstrated with a passively $Q$-switched microchip laser consisting of a Nd:YAG crystal bonded to a thin piece of $\mathrm{Cr}^{4+}$ :YAG [126], [127]. With a monolithic $\mathrm{Cr}^{4+}$ co-doped Nd:YAG laser, pulses of $290 \mathrm{ps}$ have been obtained [128]. Using active $Q$-switching, pulses as short as 115 ps have been reported [129].

From the discussion in Section II, we see that the regime of pure passive $Q$-switching requires that we reduce the cavity length substantially (4). Following this, we have passively $Q$ switched a diode-pumped Nd:LSB and Nd: $\mathrm{YVO}_{4}$ microchip laser with an A-FPSA at a center wavelength $\approx 1.06 \mu \mathrm{m}$ and achieved pulses as short as 180 ps [65] and 56 ps [66] (Fig. 17), respectively. By changing the design parameters of the saturable absorber, such as the top reflector, we can vary the pulsewidth from picoseconds to nanoseconds; by changing the pump power, we can vary the pulse repetition rate from the kilohertz to megahertz regime. Because the optical penetration depth into our typical intracavity SESAM devices is extremely short $(<1 \mu \mathrm{m})$ [83], we can maintain a very short laser cavity, allowing for minimum pulsewidths. To date, the 56-ps pulsewidths are the shortest ever produced from a $Q$-switched solid-state laser.

Our approach can also be extended to other wavelengths using different semiconductor materials. Recently, we have demonstrated a passively $Q$-switched $1.34-\mu$ m diode-pumped $\mathrm{Nd}: \mathrm{YVO}_{4}$-microchip (200- $\mu \mathrm{m}$ thick) laser. We achieved single frequency, 230-ps pulses with 100-nJ pulse energy at a repetition rate of $50 \mathrm{kHz}$, resulting in a peak power of about $450 \mathrm{~W}$ at an average power of $5 \mathrm{~mW}$ [130], [131]. As a passive $Q$-switching device, we used an MOCVD grown InGaAsP-InP A-FPSA. Shorter pulses are expected with further improvements of the A-FPSA. This is the first demonstration

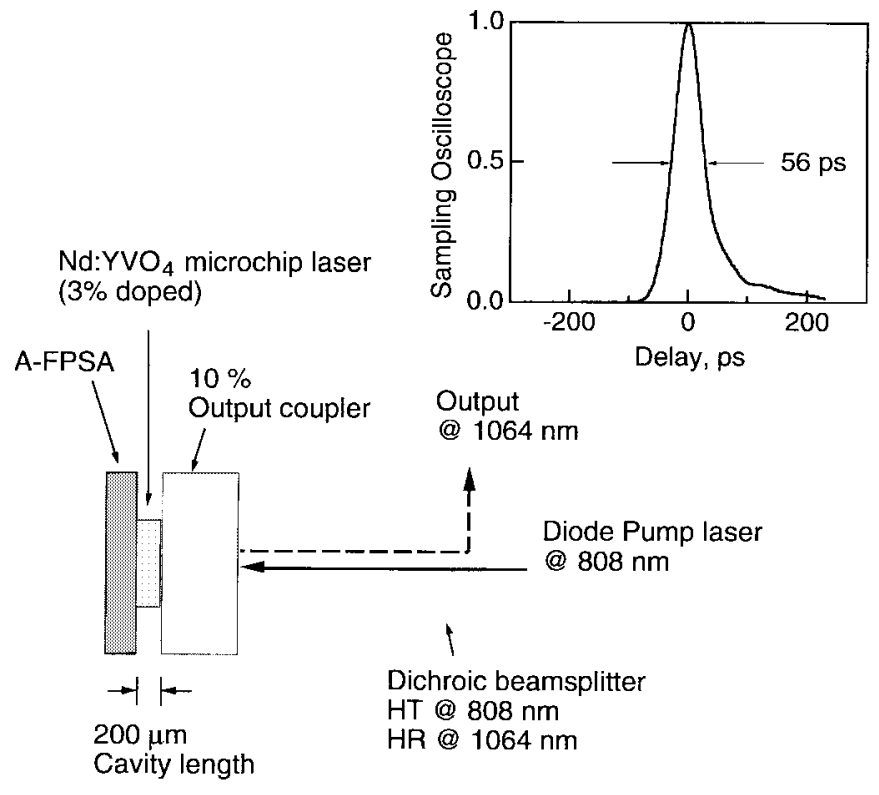

Fig. 17. Passively $Q$-switched diode-pumped $\mathrm{Nd}: \mathrm{YVO}_{4}$ microchip laser producing pulses as short as $56 \mathrm{ps}$. The shortest pulses ever produced from a $Q$-switched solid-state laser.

to our knowledge of a passively $Q$-switched microchip laser at a wavelength longer than $\approx 1 \mu \mathrm{m}$. In contrast to $\mathrm{Cr}^{4+}$ :YAG saturable absorbers, our saturable absorber devices can be adapted to longer wavelengths using different semiconductor materials. Recently, we have extended this approach to passively $Q$ switched Er:Yb:glass microchip lasers at a wavelength $\approx 1.5$ $\mu \mathrm{m}$ [131], which is important for sensing and LIDAR application where "eye-safe" wavelengths are required.

\section{CONCLUSION}

During the last six years, we observed a tremendous progress in ultrashort pulsed laser sources. Compact "realworld" picosecond and femtosecond laser systems are now a reality. This review has only considered free-space laser systems, but it is important to note that there also has been tremendous progress in mode-locked fiber lasers, and there are also similar applications of SESAM's in this area. 
Rapid progress in ultrashort pulse generation has been based on novel broad-band solid-state laser materials, and novel designs of saturable absorption and dispersion compensation. Early attempts to passively mode-lock solid-state lasers with long upper state lifetimes consistently resulted in $Q$-switched mode-locking. The main reason for this was that the absorber response time was typically in the range of $\approx 1 \mathrm{~ns}$, which reduced the saturation intensity to the point that self- $Q$ switching could not be prevented. Stable mode-locking with intracavity saturable absorbers has been achieved by varying the parameters such as response time and absorption cross section through special growth and design techniques. With SESAM's, we can benefit from control of both material and device parameters to determine the performance of the saturable absorber. We can view these as basic optoelectronic devices for ultrafast laser systems.

Soliton mode-locking provides us a new, useful model of femtosecond pulse generation. By showing that we do not need a saturable absorber with a response as fast as the pulsewidth, we have demonstrated that pulses as short as $\approx 6.5 \mathrm{fs}$ can be supported with SESAM's, and we have a new mechanism to assist in obtaining pulses below the 10 -fs level directly from the laser.

We also expect significant continued progress in the next few years to make these lasers more compact and simpler. Bulk scale optical devices will be supplanted in some applications by hybrid, quasimonolithic structures (e.g., diode-pumped $Q$ switched microchip lasers). In addition, many applications such as material processing and surgery require higher average powers $(>10-\mathrm{W} \mathrm{CW})$ and pulse energies $(>1 \mathrm{~mJ})$. Novel approaches are still needed to make such pulsed laser systems more compact. One of our key remaining research challenges will be scaling of the SESAM devices to these higher pulse energies and average powers.

In general, the capability to control both the linear and nonlinear optical properties beyond the "natural" material properties has turned out to be extremely successful, and we can only expect more progress in this direction. This design trend is also reflected in other fields, for example, quasiphase matching in nonlinear optics, bandgap engineering in semiconductor technology, and sliding filters in optical communications. In the future, similar new developments would be desirable for solid state laser materials (bandgap engineering for solid-state crystals), because ultrafast laser sources will ultimately become limited by the available material characteristics.

\section{REFERENCES}

[1] H. W. Mocker and R. J. Collins, "Mode competition and self-locking effects in a $Q$-switched ruby laser," Appl. Phys. Lett., vol. 7, pp. 270-273, 1965 .

[2] A. J. DeMaria, D. A. Stetser, and H. Heynau, "Self mode-locking of lasers with saturable absorbers," Appl. Phys. Lett., vol. 8, pp. 174-176, 1966.

[3] P. W. Smith, "Mode-locking of lasers," Proc. IEEE, vol. 58, pp. 1342-1357, 1970.

[4] H. A. Haus, "Parameter ranges for CW passive modelocking," IEEE J. Quantum Electron., vol. QE-12, pp. 169-176, 1976.

[5] U. Keller, D. A. B. Miller, G. D. Boyd, T. H. Chiu, J. F. Ferguson, and M. T. Asom, "Solid-state low-loss intracavity saturable absorber for
Nd:YLF lasers: An antiresonant semiconductor Fabry-Perot saturable absorber," Opt. Lett., vol. 17, pp. 505-507, 1992.

[6] U. Keller, T. H. Chiu, and J. F. Ferguson, "Self-starting femtosecond mode-locked Nd:glass laser using intracavity saturable absorbers," Opt. Lett., vol. 18, pp. 1077-1079, 1993.

[7] U. Keller, "Ultrafast all-solid-state laser technology," Appl. Phys. B, vol. 58, pp. 347-363, 1994.

[8] L. R. Brovelli, U. Keller, and T. H. Chiu, "Design and operation of antiresonant Fabry-Perot saturable semiconductor absorbers for modelocked solid-state lasers," J. Opt. Soc. Amer. B, vol. 12, pp. 311-322, 1995.

[9] F. X. Kärtner, L. R. Brovelli, D. Kopf, M. Kamp, I. Calasso, and U. Keller, "Control of solid-state laser dynamics by semiconductor devices," Opt. Eng., vol. 34, pp. 2024-2036, 1995.

[10] T. B. Norris, W. Sha, W. J. Schaff, X. J. Song, Z. Liliental-Weber, and E. R. Weber, "Transient absorption of low-temperature molecular-beam epitaxy grown GaAs," in Picosecond Electronics and Optoelectronics, T. C. L. Sollner and J. Shah, Eds. Washington, DC: Opt. Soc. Amer., 1991, vol. 9, pp. 244-247.

[11] U. Siegner, R. Fluck, G. Zhang, and U. Keller, "Ultrafast high-intensity nonlinear absorption dynamics in low-temperature grown gallium arsenide," Appl. Phys. Lett., vol. 69, pp. 2566-2568, 1996.

[12] E. P. Ippen, D. J. Eichenberger, and R. W. Dixon, "Picosecond pulse generation by passive modelocking of diode lasers," Appl. Phys. Lett., vol. 37, pp. 267-269, 1980.

[13] J. P. v. d. Ziel, W. T. Tsang, R. A. Logan, R. M. Mikulyak, and W. M. Augustyniak, "Subpicosecond pulses from a passively modelocked GaAs buried optical guide semiconductor lasers," Appl. Phys. Lett., vol. 39, pp. 525-527, 1981.

[14] Y. Silberberg, P. W. Smith, D. J. Eilenberger, D. A. B. Miller, A. C. Gossard, and W. Wiegmann, "Passive modelocking of a semiconductor diode laser," Opt. Lett., vol. 9, pp. 507-509, 1984.

[15] M. N. Islam, E. R. Sunderman, C. E. Soccolich, I. Bar-Joseph, N. Sauer, T. Y. Chang, and B. I. Miller, "Color center lasers passively mode locked by quantum wells," IEEE J. Quantum Electron., vol. 25, pp. 2454-2463, 1989.

[16] U. Keller, W. H. Knox, and H. Roskos, "Coupled-cavity resonant passive modelocked (RPM) Ti:Sapphire laser," Opt. Lett., vol. 15, pp. 1377-1379, 1990.

[17] U. Keller, T. K. Woodward, D. L. Sivco, and A. Y. Cho, "Coupledcavity resonant passive modelocked Nd:Yttrium lithium fluoride laser," Opt. Lett., vol. 16, pp. 390-392, 1991.

[18] H. A. Haus, U. Keller, and W. H. Knox, "A theory of coupled cavity modelocking with resonant nonlinearity," J. Opt. Soc. Amer. B, vol. 8, pp. 1252-1258, 1991.

[19] U. Keller, G. W. 'tHooft, W. H. Knox, and J. E. Cunningham, "Femtosecond pulses from a continuously self-starting passively mode-locked Ti:sapphire laser," Opt. Lett., vol. 16, pp. 1022-1024, 1991.

[20] U. Keller and T. H. Chiu, "Resonant passive modelocked Nd:YLF laser," IEEE J. Quantum Electron., vol. 28, pp. 1710-1721, 1992.

[21] U. Keller, W. H. Knox, and G. W. 'tHooft, Invited Paper, "Ultrafast solid-state modelocked lasers using resonant nonlinearities," IEEE $J$. Quantum Electron., vol. 28, pp. 2123-2133, 1992.

[22] L. F. Mollenauer and R. H. Stolen, "The soliton laser," Opt. Lett., vol. 9, pp. 13-15, 1984.

[23] K. J. Blow and B. P. Nelson, "Improved modelocking of an F-center laser with a nonlinear nonsoliton external cavity," Opt. Lett., vol. 13, pp. 1026-1028, 1988.

[24] P. N. Kean, X. Zhu, D. W. Crust, R. S. Grant, N. Landford, and W. Sibbett, "Enhanced modelocking of color center lasers," Opt. Lett., vol. 14 , pp. 39-41, 1989.

[25] E. P. Ippen, H. A. Haus, and L. Y. Liu, "Additive pulse modelocking," J. Opt. Soc. Amer. B, vol. 6, pp. 1736-1745, 1989.

[26] D. E. Spence, P. N. Kean, and W. Sibbett, " $60-\mathrm{fsec}$ pulse generation from a self-mode-locked Ti:Sapphire laser," Opt. Lett., vol. 16, pp. 42-44, 1991

[27] G. H. C. New, Opt. Commun., vol. 6, p. 188, 1974.

[28] H. A. Haus, "Theory of mode locking with a slow saturable absorber," IEEE J. Quantum Electron., vol. 11, pp. 736-746, 1975.

[29] _ "Theory of modelocking with a fast saturable absorber," J. Appl. Phys., vol. 46, pp. 3049-3058, 1975.

[30] H. A. Haus, J. G. Fujimoto, and E. P. Ippen, "Structures for additive pulse modelocking," J. Opt. Soc. Amer. B, vol. 8, pp. 2068-2076, 1991.

[31] F. X. Kärtner and U. Keller, "Stabilization of soliton-like pulses with a slow saturable absorber," Opt. Lett., vol. 20, pp. 16-18, 1995.

[32] I. D. Jung, F. X. Kärtner, L. R. Brovelli, M. Kamp, and U. Keller, 
"Experimental verification of soliton modelocking using only a slow saturable absorber," Opt. Lett., vol. 20, pp. 1892-1894, 1995.

[33] F. X. Kärtner, I. D. Jung, and U. Keller, "Soliton modelocking with saturable absorbers: Theory and experiments," this issue, pp. 540-556.

[34] J. A. Valdmanis and R. L. Fork, "Design considerations for a femtosecond pulse laser balancing self phase modulation, group velocity dispersion, saturable bbsorption, and saturable gain," IEEE J. Quantum Electron., vol. 22, pp. 112-118, 1986.

[35] R. L. Fork, C. H. B. Cruz, P. C. Becker, and C. V. Shank, "Compression of optical pulses to six femtoseconds by using cubic phase compensation," Opt. Lett., vol. 12, pp. 483-485, 1987.

[36] P. F. Moulton, "Spectroscopic and laser characteristics of $\mathrm{Ti}^{-\mathrm{Al}_{2} \mathrm{O}_{3}}$," $J$. Opt. Soc. Amer. B, vol. 3, pp. 125-132, 1986.

[37] D. K. Negus, L. Spinelli, N. Goldblatt, and G. Feugnet, "Sub-100 femtosecond pulse generation by Kerr lens modelocking in Ti:Sapphire," in Advanced Solid-State Lasers G. Dubé, L. Chase, Eds. Washington, DC: Opt. Soc. Amer., 1991, vol. 10, pp. 120-124.

[38] F. Salin, J. Squier, and M. Piché, "Modelocking of Ti:Sapphire lasers and self-focusing: A Gaussian approximation," Opt. Lett., vol. 16, pp. 1674-1676, 1991.

[39] J. Zhou, G. Taft, C.-P. Huang, M. M. Murnane, H. C. Kapteyn, and I. P. Christov, "Pulse evolution in a broad-bandwidth Ti:sapphire laser," Opt. Lett., vol. 19, pp. 1149-1151, 1994.

[40] A. Stingl, M. Lenzner, Ch. Spielmann, F. Krausz, and R. Szipöcs, "Sub-10-fs mirror-controlled Ti:sapphire laser," Opt. Lett., vol. 20, pp. 602-604, 1995.

[41] L. Xu, C. Spielmann, F. Krausz, and R. Szipöcs, "Ultrabroadband ring oscillator for sub-10-fs pulse generation," Opt. Lett., vol. 21, pp. 1259-1261, 1996.

[42] R. Szipöcs, K. Ferencz, C. Spielmann, and F. Krausz, "Chirped multilayer coatings for broadband dispersion control in femtosecond lasers," Opt. Lett., vol. 19, pp. 201-203, 1994.

[43] F. X. Kärtner, N. Matuschek, T. Schibli, U. Keller, H. A. Haus, C. Heine, R. Morf, V. Scheuer, M. Tilsch, and T. Tschudi, "Design and fabrication of double-chirped mirrors," Opt. Lett., to be published.

[44] I. D. Jung, F. X. Kärtner, N. Matuschek, D. Sutter, F. Morier-Genoud, G. Zhang, U. Keller, V. Scheuer, M. Tilsch, and T. Tschudi, "Selfstarting 6.5 fs from a KLM Ti:sapphire laser," Opt. Lett., submitted for publication.

[45] A. Baltuska, Z. Wei, M. S. Pshenichnikov, and D. A. Wiersma, "Optical pulse compression to $5 \mathrm{fs}$ at $1 \mathrm{MHz}$ repetition rate," Opt. Lett., vol. 22 , pp. 102-104, 1997

[46] M. Nisoli, S. De Silvestri, O. Svelto, R. Szipöcs, K. Ferencz, C. Spielmann, S. Sartania, and F. Krausz, Opt. Lett, vol. 22, to be published.

[47] I. P. Christov, H. C. Kapteyn, M. M. Murnane, C. P. Huang, and J. Zhou, "Space-time focusing of femtosecond pulses in a Ti:sapphire laser," Opt. Lett., vol. 20, pp. 309-311, 1995.

[48] S. T. Cundiff, W. H. Knox, E. P. Ippen, and H. A. Haus, "Frequency dependent mode size in broadband Kerr-lens mode locking," Opt. Lett., vol. 21, pp. 662-664, 1996

[49] G. Cerullo, S. De Silvestri, V. Magni, and L. Pallaro, "Resonators for Kerr-lens mode-locked femtosecond Ti:sapphire lasers," Opt. Lett., vol. 19, pp. 807-809, 1994.

[50] G. Cerullo, S. De Silvestri, and V. Magni, "Self-starting Kerr lens modelocking of a Ti:Sapphire laser," Opt. Lett., vol. 19, pp. 1040-1042, 1994.

[51] F. X. Kärtner, D. Kopf, and U. Keller, "Solitary pulse stabilization and shortening in actively mode-locked lasers," J. Opt. Soc. Amer. B, vol. 12, pp. 486-496, 1995.

[52] D. Kopf, F. Kärtner, K. J. Weingarten, and U. Keller, "Pulse shortening in a Nd:glass laser by gain reshaping and soliton formation," Opt. Lett., vol. 19, pp. 2146-2148, 1994.

[53] D. J. Kaup, "Perturbation theory for solitons in optical fibers," Phys. Rev. A, vol. 42, pp. 5689-5694, 1990.

[54] D. Kopf, F. X. Kärtner, K. J. Weingarten, and U. Keller, "Diode-pumped modelocked Nd:glass lasers using an A-FPSA," Opt. Lett., vol. 20, pp. $1169-1171,1995$

[55] I. D. Jung, F. X. Kärtner, G. Zhang, and U. Keller, "High-dynamicrange characterization of ultrashort pulses," Opt. Lett., submitted for publication.

[56] R. Fluck, I. D. Jung, G. Zhang, F. X. Kärtner, and U. Keller, "Broadband saturable absorber for $10 \mathrm{fs}$ pulse generation," Opt. Lett., vol. 21, pp. 743-745, 1996.

[57] O. E. Martinez, R. L. Fork, and J. P. Gordon, Opt. Lett., vol. 9, pp. $156-158,1984$

[58] F. Salin, P. Grangier, G. Roger, and A. Brun, "Observation of high- order solitons directly produced by a femtosecond ring laser," Phys. Rev. Lett., vol. 56, pp. 1132-1135, 1986.

[59] _ "Experimental observation of nonsymmetrical $N=2$ solitons in a femtosecond laser," Phys. Rev. Lett., vol. 60, pp. 569-571, 1988.

[60] H. Avramopoulos and G. H. C. New, "A numerical model for the study of phase effects in passive mode-locking," Opt. Commun., vol. 71, pp. 370-376, 1989 .

[61] T. Brabec, C. Spielmann, and F. Krausz, "Mode locking in solitary lasers," Opt. Lett., vol. 16, pp. 1961-1963, 1991.

[62] _ _ "Limits of pulse shortening in solitary lasers," Opt. Lett., vol 17, pp. 748-750, 1992.

[63] J. Aus der Au, D. Kopf, F. Morier-Genoud, M. Moser, and U. Keller, "60 fs pulses from a diode-pumped Nd:glass laser," Opt. Lett., vol. 22, pp. 307-309, 1997

[64] B. Braun and U. Keller, "Single frequency $Q$-switched ring laser with an antiresonant Fabry-Perot saturable absorber," Opt. Lett., vol. 20, pp. 1020-1022, 1995.

[65] B. Braun, F. X. Kärtner, U. Keller, J.-P. Meyn, and G. Huber, "Passively $Q$-switched 180 ps Nd:LSB microchip laser," Opt. Lett., vol. 21, pp. 405-407, 1996

[66] B. Braun, F. X. Kärtner, M. Moser, G. Zhang, and U. Keller, "56 ps passively $Q$-switched diode-pumped microchip laser," Opt. Lett., vol. 22, pp. 381-383, 1997.

[67] U. Keller, T. H. Chiu, and J. F. Ferguson, "Self-starting and self- $Q$ switching dynamics of a passively modelocked Nd:YLF and Nd:YAG laser," Opt. Lett., vol. 18, pp. 217-219, 1993.

[68] M. Kaminska, Z. Liliental-Weber, E. R. Weber, T. George, J. B Kortright, F. W. Smith, B.-Y. Tsaur, and A. R. Calawa, "Structural properties of As-rich GaAs grown by molecular epitaxy at low temperatures," Appl. Phys. Lett., vol. 54, pp. 1881-1883, 1989.

[69] S. Gupta, J. F. Whitaker, and G. A. Mourou, "Ultrafast carrier dynamics in III-V semiconductors grown by molecular-beam epitaxy at very low substrate temperatures," IEEE J. Quantum Electron., vol. 28, pp. 2464-2472, 1992.

[70] J. Shah, Ultrafast Spectroscopy of Semiconductors and Semiconductor Nanostructures. Berlin, Germany: Springer Verlag, 1996.

[71] D. Kopf, T. Strässle, G. Zhang, F. X. Kärtner, U. Keller, M. Moser, D. Jubin, K. J. Weingarten, R. J. Beach, M. A. Emanuel, and J. A. Skidmore, "Diode-pumped femtosecond solid state lasers based on semiconductor saturable absorbers," in Generation, Amplification, and Measurement of Ultrashort Laser Pulses III, Proc. SPIE, vol. 2701, 1996 , pp. 11-22.

[72] D. Kopf, A. Prasad, G. Zhang, M. Moser, and U. Keller, "Broadly tunable femtosecond Cr:LiSAF laser," Opt. Lett., to be published.

[73] U. Keller, "Advances in all-solid-state ultrafast lasers," in Ultrafast Phenomena X, P. F. Barbara, J. G. Fujimoto, W. H. Knox, and W. Zinth, Eds. Berlin, Germany: Springer, 1996, pp. 3-5.

[74] L. R. Brovelli, I. D. Jung, D. Kopf, M. Kamp, M. Moser, F. X. Kärtner, and U. Keller, "Self-starting soliton modelocked Ti:sapphire laser using a thin semiconductor saturable absorber," Electron. Lett., vol. 31, pp. 287-289, 1995

[75] S. Tsuda, W. H. Knox, E. A. d. Souza, W. Y. Jan, and J. E. Cunningham, "Low-loss intracavity AlAs/AlGaAs saturable Bragg reflector for femtosecond mode locking in solid-state lasers," Opt. Lett., vol. 20, pp. 1406-1408, 1995.

[76] I. D. Jung, L. R. Brovelli, M. Kamp, U. Keller, and M. Moser, "Scaling of the antiresonant Fabry-Perot saturable absorber design toward a thin saturable absorber," Opt. Lett., vol. 20, pp. 1559-1561, 1995.

[77] C. Hönninger, G. Zhang, U. Keller, and A. Giesen, "Femtosecond Yb:YAG laser using semiconductor saturable absorbers," Opt. Lett., vol. 20, pp. 2402-2404, 1995.

[78] D. Kopf, G. Zhang, R. Fluck, M. Moser, and U. Keller, "All-inone dispersion-compensating saturable absorber mirror for compact femtosecond laser sources," Opt. Lett., vol. 21, pp. 486-488, 1996.

[79] B. Braun, C. Hönninger, G. Zhang, U. Keller, F. Heine, T. Kellner, and G. Huber, "Efficient intracavity frequency doubling of a passively modelocked diode-pumped Nd:LSB laser," Opt. Lett., vol. 21, pp. 1567-1569, 1996.

[80] R. Fluck, G. Zhang, U. Keller, K. J. Weingarten, and M. Moser, "Diodepumped passively mode-locked $1.3 \mu \mathrm{m} \mathrm{Nd:YVO} 4$ and Nd:YLF lasers using semiconductor saturable absorbers," Opt. Lett., vol. 21, pp. 1378, 1996.

[81] D. Kopf, K. J. Weingarten, L. R. Brovelli, M. Kamp, and U. Keller, "Sub-50-fs diode-pumped mode-locked Cr:LiSAF with an A-FPSA," presented at the Conf. Lasers and Electro-Optics, CLEO 1995, paper CWM2. 
[82] D. Kopf, G. Zhang, M. Moser, U. Keller, M. A. Emanuel, R. J. Beach, and J. A. Skidmore, "High-power femtosecond diode-pumped Cr:LiSAF laser," presented at the Advanced Solid-State Lasers Conference, Jan. 27-29, 1997, paper MF1; also in Opt. Lett., vol. 22, submitted for publication

[83] L. R. Brovelli and U. Keller, "Simple analytical expressions for the reflectivity and the penetration depth of a Bragg mirror between arbitrary media," Opt. Commun., vol. 116, pp. 343-350, 1995.

[84] S. Tsuda, W. H. Knox, E. A. d. Souza, W. Y. Jan, and J. E. Cunningham, "Femtosecond self-starting passive modelocking using an AlAs/AlGaAs intracavity saturable Bragg reflector," in CLEO 1995, paper CWM6, p. 254.

[85] B. G. Kim, E. Garmire, S. G. Hummel, and P. D. Dapkus, "Nonlinear Bragg reflector based on saturable absorption," Appl. Phys. Lett., vol. 54, pp. 1095-1097, 1989.

[86] M. Ramaswamy-Paye and J. G. Fujimoto, "Compact dispersioncompensating geometry for Kerr-lens mode-locked femtosecond lasers,' Opt. Lett., vol. 19, pp. 1756-1758, 1994.

[87] D. Kopf, G. J. Spühler, K. J. Weingarten, and U. Keller, "Mode-locked laser cavities with a single prism for dispersion compensation," Appl. Opt., vol. 35, pp. 912-915, 1996.

[88] R. L. Fork, O. E. Martinez, and J. P. Gordon, "Negative dispersion using pairs of prisms," Opt. Lett., vol. 9, pp. 150-152, 1984.

[89] K. Ferencz and R. Szipöcs, "Recent developments of laser optical coatings in Hungary," Opt. Eng., vol. 32, pp. 2525-2538, 1993.

[90] R. Szipöcs, A. Stingl, C. Spielmann, and F. Krausz, "Chirped dielectric mirrors for dispersion control in femtosecond laser systems," F. W Wise, C. P. J. Bartys, Eds., in Generation, Amplification, and Measurement of Ultrashort Laser Pulses II, Proc. SPIE Proc. 1995, vol. 2377, pp. 11-12.

[91] F. Gires and P. Tournois, "Interferometer utilisable pour la compression d'impulsions lumineuses modulees en frequence," C.R.Acad.Sci.Paris, vol. 258, pp. 6112-6115, 1964.

[92] D. A. B. Miller, D. s. Chemla, T. C. Damen, A. C. Gossard, W Wiegmann, T. H. Wood, and C. A. Burrus, Phys. Rev. Lett., vol. 53, p. $2173,1984$.

[93] _ _ "Electric field dependence of optical absorption near the band gap of quantum well structures," Phys. Rev. B, vol. 32, pp. 1043-1060, 1985.

[94] D. A. B. Miller, J. S. Weiner, and D. S. Chemla, "Electric Field Dependence of Linear Optical Properties in Quantum Well Structures: Waveguide Electroabsorption and Sum Rules," IEEE J. Quantum Electron., vol. 22, pp. 1816-1830, 1986.

[95] A. M. Fox, D. A. B. Miller, G. Livescu, J. E. Cunningham, J. E. Henry, and W. Y. Jan, "Exciton saturation in electrically biased quantum wells," J. Appl. Phys. Lett., vol. 57, p. 2315, 1990.

[96] T. Sizer, II, T. K. Woodward, U. Keller, K. Sauer, T.-H. Chiu, D. L. Sivco, and A. Y. Cho, "Measurement of carrier escape rates, exciton saturation intensity, and saturation density in electrically biased multiple-quantum-well modulators," IEEE J. Quantum Electron. vol. 30 , pp. 399-407, 1994.

[97] L. R. Brovelli, M. Lanker, U. Keller, K. W. Goossen, J. A. Walker, and J. E. Cunningham, "An antiresonant Fabry-Perot quantum well modulator to actively mode-lock and synchronize solid-state lasers," Electron. Lett., vol. 31, pp. 381-382, 1995.

[98] S. A. Payne, L. L. Chase, L. K. Smith, W. L. Kway, and H. Newkirk, "Laser performance of $\mathrm{LiSrAlF}_{6}: \mathrm{Cr}^{3+}$," J. Appl. Phys., vol. 66, pp. 1051-1056, 1989.

[99] S. A. Payne, L. L. Chase, H. W. Newkirk, L. K. Smith, and W. F. Krupke, "Cr:LiCAF: A promising new solid-state laser material," IEEE J. Quantum Electron., vol. 24, pp. 2243-2252, 1988.

[100] H. S. Wang, P. L. K. Wa, J. L. Lefaucheur, B. H. T. Chai, and A. Miller, "CW and self-mode-locking performance of a red pumped Cr:LiSCAF laser," Opt. Commun., vol. 110, pp. 679-688, 1994.

[101] L. K. Smith, S. A. Payne, W. L. Kway, L. L. Chase, and B. H. T. Chai, "Investigation of the laser properties of $\mathrm{Cr}_{3+}: \mathrm{LiSrGaF} 6$," IEEE J. Quantum Electron., vol. 28, pp. 2612-2618, 1992.

[102] M. Stalder, M. Bass, and B. H. T. Chai, "Thermal quenching of fluorescence in chromium-doped fluoride laser crystals," J. Opt. Soc. Amer. B, vol. 9, pp. 2271-2273, 1992.

[103] P. M. W. French, R. Mellish, J. R. Taylor, P. J. Delfyett, and L. T. Florez, "All-solid-state diode-pumped modelocked Cr:LiSAF laser," Electron. Lett., vol. 29, pp. 1262-1263, 1993.

[104] P. M. W. French, R. Mellish, and J. R. Taylor, "Modelocked all-solidstate diode-pumped Cr:LiSAF laser," Opt. Lett., vol. 18, pp. 1934-1936, 1993
[105] P. M. Mellish, P. M. W. French, J. R. Taylor, P. J. Delfyett, and L. T. Florez, "All-solid-state femtosecond diode-pumped Cr:LiSAF laser," Electron. Lett., vol. 30, pp. 223-224, 1994.

[106] D. Kopf, K. J. Weingarten, L. Brovelli, M. Kamp, and U. Keller, "Diode-pumped sub-100-fs passively mode-locked Cr:LiSAF using an A-FPSA," presented at CLEO 1994, paper CPD22.

[107] _ "Diode-pumped 100-fs passively mode-locked Cr:LiSAF using an A-FPSA," Opt. Lett., vol. 19, pp. 2143-2145, 1994.

[108] S. Tsuda, W. H. Knox, and S. T. Cundiff, "High efficiency diode pumping of a saturable Bragg reflector-mode-locked Cr:LiSAF femtosecond laser," Appl. Phys. Lett., vol. 69, pp. 1538-1540, 1996.

[109] M. J. P. Dymott and A. I. Ferguson, "Self-mode-locked diode-pumped Cr:LiSAF laser producing $34-\mathrm{fs}$ pulses at $42-\mathrm{mW}$ average power," Opt. Lett., vol. 20, pp. 1157-1159, 1995.

[110] R. Mellish, N. P. Barry, S. C. W. Hyde, R. Jones, P. M. W. French, J. R. Taylor, C. J. v. d. Poel, and A. Valster, "Diode-pumped Cr:LiSAF all-solid-state femtosecond oscillator and regenerative amplifier," Opt. Lett., vol. 20, pp. 2312-2314, 1995.

[111] D. Kopf, J. Aus der Au, U. Keller, G. L. Bona, and P. Roentgen, "A 400-mW continous-wave diode-pumped Cr:LiSAF laser based on a power-scalable concept," Opt. Lett., vol. 20, pp. 1782-1784, 1995.

[112] D. Kopf, U. Keller, M. A. Emanuel, R. J. Beach, and J. A. Skidmore, "A 1.1-W CW Cr:LiSAF laser pumped by a 1-cm diode-array," Opt. Lett., vol. 22, pp. 99-101, 1997.

[113] S. Basu and R. L. Byer, "Continuous-wave mode-locked Nd:glass laser pumped by a laser diode," Opt. Lett., vol. 13, pp. 458-460, 1988.

[114] C. Spielmann, F. Krausz, T. Brabec, E. Wintner, and A. J. Schmidt, "Femtosecond passive modelocking of a solid-state laser by dispersively balanced nonlinear interferometer," Appl. Phys. Lett., vol. 58, pp. 2470-2472, 1991.

[115] P. Lacovara, H. K. Choi, C. A. Wang, R. L. Aggarwal, and T. Y. Fan, "Room-temperature diode-pumped Yb:YAG laser," Opt. Lett., vol. 16 , pp. 1089-1091, 1991.

[116] T. Y. Fan, "Heat Generation in Nd:YAG and Yb:YAG," IEEE $J$. Quantum Electron., vol. 29, pp. 1457-1459, 1993.

[117] U. Brauch, A. Giesen, M. Karszewski, C. Stewen, and A. Voss, "Multiwatt diode-pumped Yb:YAG thin disk laser continuously tunable between 1018 and 1053 nm," Opt. Lett., vol. 20, pp. 713-715, 1995.

[118] R. Allen and L. Esterowitz, "CW tunable ytterbium YAG laser pumped by titanium sapphire," Electron. Lett., vol. 31, pp. 639-641, 1995.

[119] S. R. Henion, P. A. Schulz, "Yb:YAG laser: Mode-locking and highpower operation," in CLEO 1992, p. 540, paper CThQ2.

[120] C. J. Flood, D. R. Walker, and H. M. van Driel, "The effect of spatial hole burning in a mode-locked, diode end-pumped, Nd:YAG laser," Opt. Lett., vol. 20, pp. 58-60, 1995.

[121] B. Braun, K. J. Weingarten, F. X. Kärtner, and U. Keller, "Continuouswave mode-locked solid-state lasers with enhanced spatial hole-burning, Part I: Experiments,” Appl. Phys. B, vol. 61, pp. 429-437, 1995.

[122] F. X. Kärtner, B. Braun, and U. Keller, "Continuous-wave-mode-locked solid-state lasers with enhanced spatial hole-burning, Part II: Theory," Appl. Phys. B, vol. 61, pp. 569-579, 1995.

[123] S. Spälter, M. Böhm, B. Mikulla, A. Sizmann, G. Leuchs, R. Fluck, I. D. Jung, G. Zhang, and U. Keller, "Self-starting soliton modelocked femtosecond Cr:YAG laser using an antiresonant Fabry-Perot saturable absorber," presented at CLEO Europe, 1996, Postdeadline Paper.

[124] K. J. Weingarten, U. Keller, T. H. Chiu, and J. F. Ferguson, "Passively mode-locked diode-pumped solid-state lasers using an antiresonant Fabry-Perot saturable absorber," Opt. Lett., vol. 18, pp. 640-642, 1993.

[125] Prof. R. Wallenstein, University of Kaiserslautern, Germany, private communication, 1996.

[126] J. J. Zayhowski and C. Dill III, "Diode-pumped passively $Q$-switched picosecond microchip lasers," Opt. Lett., vol. 19, pp. 1427-1429, 1994.

[127] J. J. Zayhowski, J. Ochoa, and C. Dill, III, "UV generation with passively $Q$-switched picosecond microchip lasers," presented at CLEO 1995 , p. 139 , paper CTuM2.

[128] P. Wang, S.-H. Zhou, K. K. Lee, and Y. C. Chen, "Picosecond laser pulse generation in a monolithic self- $Q$-switched solid-state laser," Opt. Commun., vol. 114, pp. 439-441, 1995.

[129] J. J. Zayhowski and C. Dill III, "Coupled cavity electro-optically $Q$ switched Nd: $\mathrm{YVO}_{4}$ microchip lasers," Opt. Lett., vol. 20, pp. 716-718, 1995.

[130] R. Fluck, B. Braun, U. Keller, E. Gini, and H. Melchior, "Passively $Q$ switched $1.34 \mu \mathrm{m} \mathrm{Nd}: \mathrm{YVO}_{4}$ microchip laser," Advanced Solid-State Lasers 1997, paper WD5.

[131] _ "Passively $Q$-switched microchip lasers at $1.3 \mu \mathrm{m}$ and $1.5 \mu \mathrm{m}$," presented at CLEO 1997. 


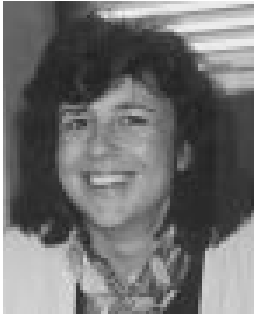

Ursula Keller (M'89) was born in Zug, Switzerland, in 1959. She received the "Diplom" in physics from the Federal Institute of Technology (ETH) Zürich, Switzerland, in 1984, and the M.S. and Ph.D. degree in applied physics from Stanford University, Stanford, CA, in 1987 and 1989, respectively.

Her Ph.D. research was in optical probing of charge and voltage in GaAs integrated circuits and in low-noise ultrafast laser systems. From late 1984 to 1985 , she worked on optical bistability at HeriotWatt University, Edinburgh, Scotland. In 1989, she joined AT\&T Bell Laboratories, Holmdel, NJ, as a Member of Technical Staff where she conducted research on photonic switching, ultrafast laser systems, and semiconductor spectroscopy. Since March 1993, she is an associate Professor in the Physics Department at the Swiss Federal Institute of Technology (ETH) in Zürich, Switzerland. Her current research interests are in ultrafast lasers, spectroscopy, local probes and novel devices for applications in optical information processing, communication and medicine. She published more than 70 journal papers, one book chapter and holds five patents.

Prof. Keller is a member of the Optical Society of America (OSA), the European Physical Society (EPS), the Swiss Physical Society (SPS), and the Swiss Academy of Technical Sciences (SATW). During 1985 to 1986, she was a Fulbright Fellow and in 1987 to 1988, she received an IBM Predoctoral Fellowship.

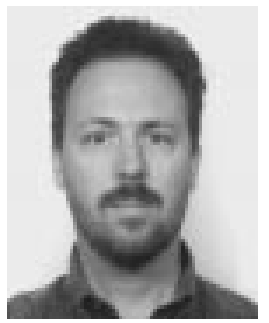

Kurt J. Weingarten (S'82-M'88) was born January 30, 1961, in St. Petersburg, FL. He received the B.S degree from the Georgia Institute of Technology in 1983, and the M.S. degree in 1985 and Ph.D. degree in 1987 from Stanford University, all in electrical engineering.

His $\mathrm{Ph} . \mathrm{D}$. work involved developing an electrooptic sampling system to test GaAs integrated circuits. From 1987 to 1993 , he worked at Lightwave Electronics as a group leader, further developing an electro-optic sampling system, then a diode-pumped mode-locked laser product series. In 1993, he moved to Zurich, Switzerland, working as a research associate in the Ultrafast Laser Physics Group at the Swiss Federal Institute of Technology (ETH) and as an independent laser consultant. In 1995, he founded the company Time-Bandwidth Products to commercialize recent developments in the area of diode-pumped ultrafast laser systems. He is a member of the Optical Society of America.

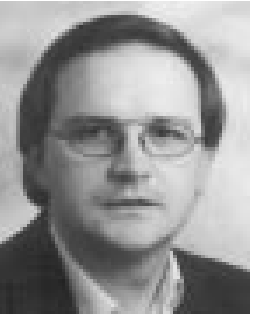

Franz X. Kärtner was born in Cham, Germany, in 1961. He received the Diploma degree in 1986 and the Ph.D. degree in 1989 in electrical engineering from the Technical University Munich.

From 1991 to 1993, he was a Feodor-Lynen Research Fellow of the Humboldt Foundation at Massachusetts Institute of Technology, USA. Since 1993, he is with the Institute of Quantum Electronics, Swiss Federal Institute of Technology (ETH) Zürich, Switzerland. He carried out research on noise in electronic circuits and optical devices, generation of squeezed states in the microwave and optical domain, quantum optics and laser physics in general. He is currently focused on the dynamics of mode-locked lasers.

Dr. Kärtner is a member of the German Physical Society and the Optical Society of America.

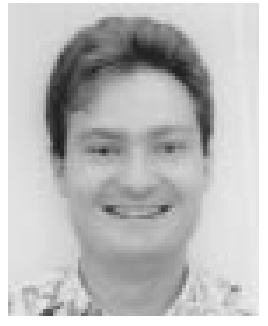

Daniel Kopf received the Diploma degree in physics in 1992 and the Ph.D. degree in 1996 from the Swiss Federal Institute of Technology (ETH) in Zurich, Switzerland.

His main research interests are in the field of compact ultrafast and diode-pumped lasers, and semiconductor physics.

Dr. Kopf is a member of the Optical Society of America

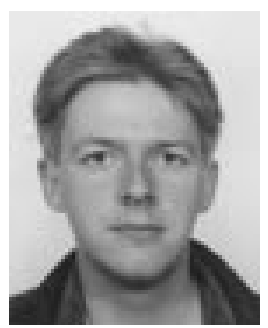

Bernd Braun was born in Bamberg, Germany, 1965. He studied physics at the Ruprecht-Karls Universität Heidelberg and the Imperial College in London and received the diploma degree in 1993, and is currently pursuing the Ph.D. degree at the Institute of Quantum Electronics, ETH Zürich.

His current research interest is focused on passively $Q$-switched diode pumped microchip lasers.

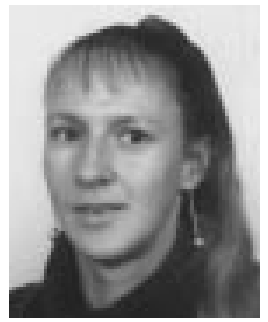

Isabella D. Jung was born in Munich, Germany 1968. She studied Physics at the LudwigMaximilian-University Munich, where she received the diploma degree in 1993 and is currently pursuing the Ph.D. degree at the Institute of Quantum Electronics, ETH Zurich.

Her current interest is focused on ultrashort pulse generation.

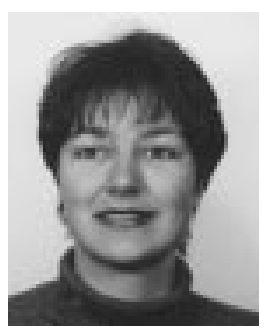

Regula Fluck received the diploma degree in physics from the Swiss Federal Institute of Technology (ETH), Zürich, Switzerland, in 1993 and is currently pursuing the $\mathrm{Ph} . \mathrm{D}$. degree at the Institute of Quantum Electronics, ETH, Zürich.

Her current interest is focused on diode pumped microchip lasers in the infrared.

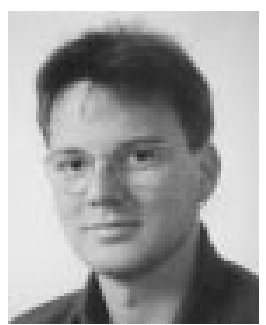

Clemens Hönninger received the diploma degree in physics from the University of Heidelberg, Germany, in 1994 and is currently pursuing the Ph.D. degree at the Institute of Quantum Electronics, ETH, Zürich.

His current research interests are in high peak power laser sources. 


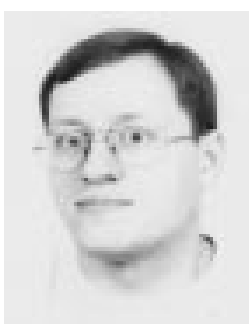

Nicolai Matuschek was born in Biberach/Riß, Germany, in 1968. He received the diploma degree in physics from the University Ulm, in 1995and is currently pursuing the Ph.D. degree at the Institute of Quantum Electronics, Swiss Federal Institute of Technology (ETH) Zürich, Switzerland.

After finishing the diploma degree in the field of theoretical polymer physics, he changed his research interests to laser physics, where he is currently focused on the theory of mode-locked solid-state lasers.

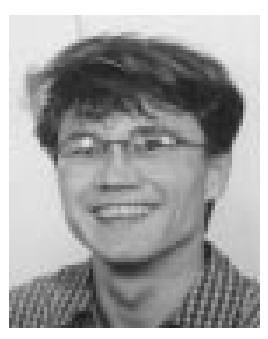

Juerg Aus der Au received the diploma degree in physics from the Swiss Federal Institute of Technology (ETH) in Zürich, Switzerland, in 1996 and is currently pursuing the Ph.D. degree at the same institute where his main research interests are focused on compact femtosecond laser sources. 